Review

\title{
Functional Nanostructures for Effective Delivery of Small Interfering RNA Therapeutics
}

\author{
Cheol Am Hong ${ }^{1}$ and Yoon Sung Nam ${ }^{1,2,3}$ \\ 1. Department of Biological Sciences, Korea Advanced Institute of Science and Technology (KAIST), 291 Daehak-ro, Yuseong-gu, Daejeon, \\ 305-701, Republic of Korea; \\ 2. Department of Materials Science and Engineering, Korea Advanced Institute of Science and Technology (KAIST), 291 Daehak-ro, \\ Yuseong-gu, Daejeon, 305-701, Republic of Korea; \\ 3. KAIST Institute for NanoCentury and BioCentury, Korea Advanced Institute of Science and Technology (KAIST), 291 Daehak-ro, \\ Yuseong-gu, Daejeon, 305-701, Republic of Korea.
}

$\square$ Corresponding author: Yoon Sung Nam, E-mail: yoonsung@kaist.ac.kr; Tel.: +82-42-350-3311; Fax: +82-42-350-3310.

() Ivyspring International Publisher. This is an open-access article distributed under the terms of the Creative Commons License (http://creativecommons.org/ licenses/by-nc-nd/3.0/). Reproduction is permitted for personal, noncommercial use, provided that the article is in whole, unmodified, and properly cited.

Received: 2014.01.04; Accepted: 2014.02.23; Published: 2014.09.19

\begin{abstract}
Small interfering RNA (siRNA) has proved to be a powerful tool for target-specific gene silencing via RNA interference (RNAi). Its ability to control targeted gene expression gives new hope to gene therapy as a treatment for cancers and genetic diseases. However, siRNA shows poor pharmacological properties, such as low serum stability, off-targeting, and innate immune responses, which present a significant challenge for clinical applications. In addition, siRNA cannot cross the cell membrane for RNAi activity because of its anionic property and stiff structure. Therefore, the development of a safe, stable, and efficient system for the delivery of siRNA therapeutics into the cytoplasm of targeted cells is crucial. Several nanoparticle platforms for siRNA delivery have been developed to overcome the major hurdles facing the therapeutic uses of siRNA. This review covers a broad spectrum of non-viral siRNA delivery systems developed for enhanced cellular uptake and targeted gene silencing in vitro and in vivo and discusses their characteristics and opportunities for clinical applications of therapeutic siRNA.
\end{abstract}

Key words: gene delivery; gene silencing; nanoparticles; non-viral vectors; small interfering RNA (siRNA).

\section{Introduction}

Gene silencing by RNA interference (RNAi) was first identified in a worm (C. elegans) in 1998 as a response to long double-stranded RNA (dsRNA). ${ }^{1}$ This finding has been extended to plants, Drosophila, and fungi. ${ }^{2-4}$ However, in mammalian cells, the introduction of long dsRNA activates anti-viral defense responses and nonspecific gene silencing though RNAi. ${ }^{5}$ The RNAi was considered as a highly conserved natural mechanism that controls the gene expression triggered by a long dsRNA in the cytoplasm. ${ }^{6}$ It was revealed that long dsRNA is processed into short, uniform RNA fragments with $21 \sim 25$ base pairs (bp) by an RNase type-III enzyme, Dicer. ${ }^{7.8}$ The
RNA fragments are incorporated into the RNA-induced silencing complex (RISC) and selectively retrain one strand of the siRNA duplex. The RISC scans and degrades a messenger RNA (mRNA) complementary to the RNA loaded within the RISC, resulting in sequence-specific gene silencing, after which it is recycled for several rounds. ${ }^{9}$ In 2001, RNAi-mediated gene silencing in mammalian cells was observed by short and synthetic dsRNA, known as small interfering RNA (siRNA), which initiated a new promising approach for therapeutic applications of RNAi. ${ }^{10,11}$ Unlike long dsRNA with over $100 \mathrm{bp}$, synthetic siRNA has $21 \sim 23 \mathrm{bp}$ with a 2-nucleotide 
overhang at both of the $3^{\prime}$ ends. In addition, siRNA is incorporated into RISC without a Dicer processing step. ${ }^{12}$ Synthetic siRNA has attracted much attention because it can easily be designed and customized for any gene.

Despite the increasing interest in siRNA-mediated gene silencing as a therapeutic strategy, there remain many critical hurdles for practical applications, including rapid enzymatic degradation and poor cellular uptake of siRNA. ${ }^{13,14}$ The development of effective siRNA delivery systems that can safely escort siRNA into the cytoplasm of targeted cells for successful siRNA therapy is crucial. To this end, viruses (e.g., adenovirus, retrovirus, and lentivirus) have been studied as potential vectors for siRNA delivery owing to their ability specifically to attach and transport their own genomic materials into cells. ${ }^{15-17}$ Although such viral vectors have relatively high transfection efficiency, their clinical application has been restricted by the potential risks of mutation, inflammation, and immune responses. ${ }^{18}$ These increasing concerns have led to the development of synthetic non-viral vectors.

A variety of synthetic vectors based on cationic polymers, peptides, and lipids have been suggested to form compact nano-sized complexes with a hydrodynamic diameter of about $200 \mathrm{~nm}$ upon contact with polyanionic nucleic acids. ${ }^{19-21}$ These polyelectrolyte complexes have a net positive charge, which can increase the chance of interaction with negatively charged cell membranes and facilitate cellular uptake via endocytosis. The structural stability of the complexes depends upon the electrostatic interaction between the nucleic acids and cationic carriers in an aqueous solution. In general, plasmid DNA can be effectively condensed with cationic carriers to produce stable nanoscale complexes. ${ }^{22}$ This approach is applied to the siRNA delivery through originally developed for plasmid DNA delivery. Unlike plasmid DNA, siRNA has a stiff structure and relatively low spatial charge density, making it difficult to formulate compact and condensed siRNA complexes. ${ }^{23,24}$ Un- stable, loose siRNA complexes can be readily attacked by enzymes in biological fluids, resulting in the rapid degradation of siRNA before its arrival at a target site. ${ }^{25}$ To achieve enhanced structural stability of the siRNA complexes, the use of an excess amount of cationic carriers has been attempted. However, this can increase the degree of nonspecific cytotoxicity. ${ }^{26,27}$ Therefore, the effective delivery of siRNA into the cytoplasm of targeted cells is essential for successful siRNA-based therapeutic applications. The current preclinical trials of RNAi therapeutics are summarized in Table 3.

In this review article, we detail the recent progress in the development of synthetic non-viral siRNA delivery systems, including siRNA-polymer conjugates, siRNA-encapsulated liposomes, inorganic nanoparticles, and engineered siRNA-based structures with RNAi activity.

\section{2. siRNA-Polymer Conjugates}

One of the attractive strategies for the effective intracellular delivery of siRNA is the chemical conjugation of siRNA to synthetic polymers. The terminal nucleotide modification of siRNA provides a convenient means of coupling siRNA with a variety of polymers. In general, the modification of sense-strand siRNA ends is preferred for siRNA-polymer conjugates because an antisense strand plays a critical role in guiding the cleavage of a target mRNA for RNAi activity. ${ }^{28,29}$ The siRNA-polymer conjugates linked via biodegradable bonds (e.g., reducible and acid-sensitive bonds) can be readily dissociated, releasing intact siRNA molecules into the cytoplasm following the cellular uptake step. In contrast, non-degradable siRNA conjugates are processed by endonuclease Dicer to regenerate biologically active siRNA species in the cell (Figure 1). Depending on the physicochemical properties of the polymer, siRNA-polymer conjugates show unique and outstanding delivery characteristics in vitro and in vivo (Table 1).

Table I. Summary of siRNA-polymer conjugates used in siRNA delivery systems.

\begin{tabular}{|c|c|c|c|c|c|c|}
\hline siRNA conjugates & Carriers & Diameter (nm) & Zeta potential $(\mathrm{mV})$ & Target genes & Target cells & Ref. \\
\hline PEG & KALA peptides, bPEI (25 kDa) & $98.7 \pm 5.1$ & Not shown & VEGF & DLD-1, PC-3 & $30 \sim 32$ \\
\hline 6-arm PEG & KALA peptides & $178 \pm 32$ & $+21.5 \pm 1.0$ & GFP & MDA-MB-435 & 33 \\
\hline oligospermine & Not used & & & Luciferase & A549 & 37 \\
\hline Lipids- oligospermine & Not used & $15 \sim 50$ & Not shown & Luciferase & A549 & 38 \\
\hline PBAVE-g-PEG, NAG, & Not used & $10 \pm 2$ & Not shown & ApoB, PPARa & Hepatocytes & 39 \\
\hline PLL-g-PEG, DMMAn-Mel & Not used & $80 \sim 300$ & Not shown & Luciferase & Neuro-2A & 40 \\
\hline PLGA & LPEI (2.5 and $25 \mathrm{kDa})$ & $30.8 \pm 7.7$ & $+20.2 \pm 7.9$ & GFP & MDA-MB-435 & 45 \\
\hline Hyaluronic acid & LPEI (25 kDa) & $250 \sim 300$ & $-25 \sim-20$ & Luciferase & MDA-MB-231, MCF7 & 46 \\
\hline Dextran & LPEI (25 kDa) & $122.8 \pm 0.42$ & $+15.07 \pm 0.38$ & GFP & A549, HeLa & 47 \\
\hline Dextran & Not used & $350.7 \pm 44.4$ & Not shown & Luciferase & PC-3, LnCap & 48 \\
\hline
\end{tabular}




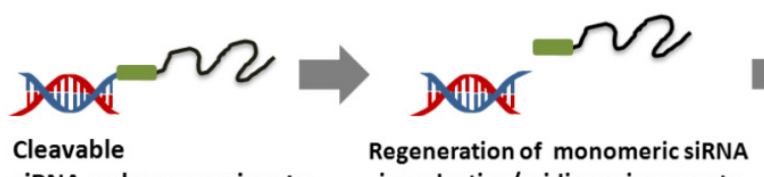

siRNA-polymer conjugate
Regeneration of monomeric siRNA
in reductive/acidic environments

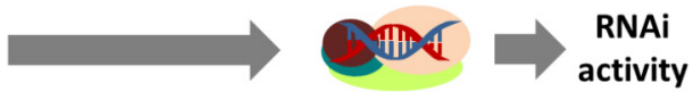

RISC (RNA-induced silencing complex)
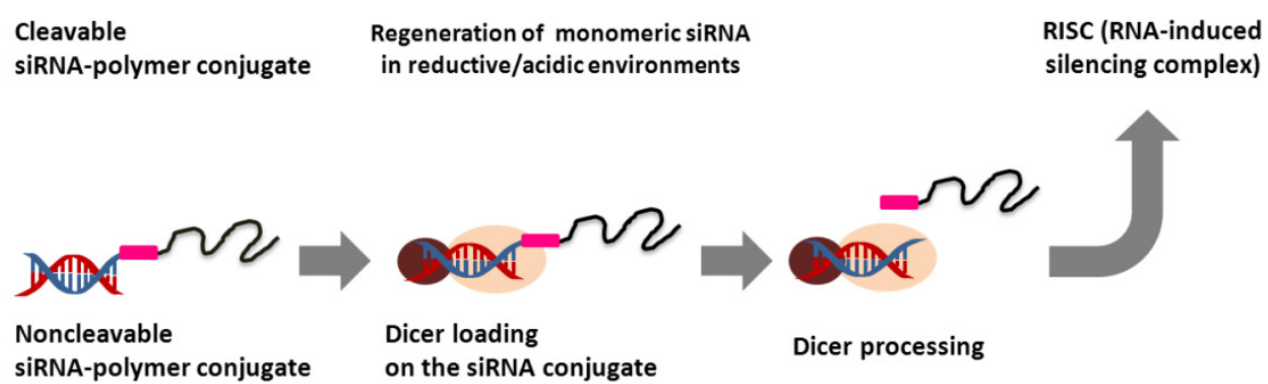

Figure I. Comparison of the intracellular processing of cleavable and non-cleavable siRNA-polymer conjugates for RNAi activity.

\section{I. PEG conjugated siRNA delivery systems}

Polyethyleneglycol (PEG) has been widely used in gene delivery due to its steric stabilization effects, biocompatibility, and anti-fouling properties. The siRNA-PEG conjugate linked with disulfide bonds was developed for systemic siRNA delivery (Figure 2a). ${ }^{30-32}$ The siRNA-PEG conjugate was electrostatically complexed with cationic carriers (e.g., branched polyethylenimine (bPEI, $25 \mathrm{kDa}$ ) and KALA peptides) to form stable polyelectrolyte complex (PEC) micelles. These micelles have a core/shell structure composed of a siRNA/polycation complex in the core and a PEG corona in the shell. The PEG corona chain prevents the access of nucleases to the siRNA loaded within the core, thus improving the blood circulation of siRNA. The stability of the siRNA encapsulated into the PEC micelles was maintained for more than $48 \mathrm{~h}$ in a $50 \%$ serum-containing medium, while the siRNA of the siRNA/bPEI complexes without the PEG shell completely degraded after $8 \mathrm{~h}$ under the same condition. In addition, the siRNA-PEG conjugate showed a significant inhibition of vascular endothelial growth factor (VEGF) expression in tumors and suppressed the tumor growth following intratumoral and systemic injections. Recently, it was also reported that a six-armed PEG derivative was co-decorated with siRNA and a cell-penetrating peptide, Hph1, via a disulfide bond for enhanced cellular uptake and gene silencing. ${ }^{33}$ The resultant six-armed PEG-siRNAHph1 conjugate was composed of siRNA and Hph1 at a ratio of approximately $3: 1$. This conjugate interacted with KALA peptides to form stable complexes with a diameter of $178 \pm 32 \mathrm{~nm}$ and a zeta potential of $21.5 \pm$ $1.0 \mathrm{mV}$. It was conceivable that the conjugated siRNA segments were incorporated into the core of the PEC micelles and that multiple PEG chains and Hph1 were exposed to their surface. The level of green fluorescent protein (GFP) fluorescence in the cells treated with the six-armed PEG-siRNA-Hph1/KALA micelles decreased by $69 \%$, which indicates significantly more efficient gene silencing as compared to that of siRNA/KALA complexes and six-armed PEG-siRNA/KALA micelles. The same research groups also explored the effects of the conjugation position in non-cleavable siRNA-PEG conjugates on the efficiency of gene silencing. ${ }^{34}$ They prepared siRNA-PEG conjugates with different conjugation sites at the $3^{\prime}$ end and $5^{\prime}$ end of the sense and antisense strands of siRNA. It was found that the PEGylation sites of siRNA were not critical factors and did not significantly affect the RNAi activity. However, the conjugation of siRNA to PEG at the $3^{\prime}$ end of the antisense strand elicited an immune response compared to cleavable siRNA-PEG conjugates. In another study, siRNA-PEG conjugates were functionalized with lactose $^{35}$ and folic acid ${ }^{36}$ as targeting ligands at the distal end of PEG for targeted siRNA delivery. The cell-targetable PEC micelles strongly interacted with the receptors on the surface of the targeted cells, resulting in enhanced cellular uptake and gene silencing at low doses of siRNA.

\subsection{Self-delivering siRNA conjugates without the help of cationic carriers}

Cationic polymers have also been linked to the end of siRNA for use as a self-delivering siRNA conjugates (Figure $2 b$ ). The cationic siRNA conjugates do not require complexation with polymeric carriers for efficient siRNA delivery into cells and more than 10 times smaller than a typical polyelectrolyte complex $(\sim 200 \mathrm{~nm})$. For instance, Nothisen et al. developed cationic oligospermine-siRNA conjugates by grafting the appropriate number of cationic spermine units to the end of siRNA for carrier-free siRNA delivery. ${ }^{37}$ They were composed of 30 spermine molecules at the $5^{\prime}$ end of sense strands, which made the net charge of siRNA positive. Confocal microscopy analysis indicated that a fluorescently labeled cationic siRNA conjugates showed a robust and diffuse staining pattern in the cytoplasm. A dose-dependent knockdown of luciferase expression was observed without the 
help of a cationic carrier. In addition, lipids (e.g., C12, C14, and cholesterol) were also conjugated to the end of oligospermine in cationic siRNA conjugates. ${ }^{38}$ Transmission electron microscopy (TEM) image of the lipid-cationic siRNA conjugates showed mostly round shaped micellar structures (< about $50 \mathrm{~nm}$ ) compared to cationic siRNA conjugates with $4 \sim 6 \mathrm{~nm}$ spots. However, there was no significant difference in gene silencing efficiency between them. Multiple different elements such as targeted ligands, endosomal escape moieties, and anti-fouling agents have also been grafted to the end of siRNA for in vivo self-delivery of siRNA. Rozema et al. developed a multifunctional self-delivering siRNA conjugates called dynamic siRNA polyconjugates. ${ }^{39}$ The chemical crosslinkers were used to reversibly graft PEG as an anti-fouling agent and $\mathrm{N}$-acetylgalactosamine (NAG) as a hepatic targeting ligand onto an endosomolytic polymer, poly(vinyl ether) (PBAVE), after which an siRNA was conjugated to the end of modified PBAVE via a disulfide bond. The resultant siRNA polyconjugates had a negatively charged surface, high solubility, excellent dispersion stability under physiological conditions, and a hydrodynamic diameter of $10 \pm 2 \mathrm{~nm}$. They were engaged with galactose-specific receptors on hepatocytes and taken into the cells via receptor-mediated endocytosis. In the endosome, the decreased $\mathrm{pH}$ induced the dissociation of PEG and NAG from siRNA polyconjugates, activating the endosmolytic capability of PBAVE and release of the siRNA-PBAVE conjugates into the cytoplasm. The released siRNA conjugates were degraded to generate free siRNA via the cleavage of their disulfide bonds. This exhibited the effective knockdown (about $80 \sim 90$ $\%$ ) of two endogenous genes, $A p o B$ and PPAR $\alpha$, in a mouse liver after an i.v. injection. Interestingly, the gene-silencing effect lasted up to 10 days and returned to near the control level by day 15 without cytotoxicity or adverse inflammatory responses. Meyer et al. also demonstrated bioresponsive and endosomolytic siRNA polyconjugates based on a PEG-modified poly-L-lysine (PLL) backbone coupled with siRNA and dimethylmaleic anhydride masked melittin (DMMAn-Mel) for endosomal release. ${ }^{40}$ The siRNA polyconjugates showed excellent structural stability against anionic heparins but were readily disassembled into monomeric siRNA under reducing conditions, enabling siRNA-mediated gene silencing.

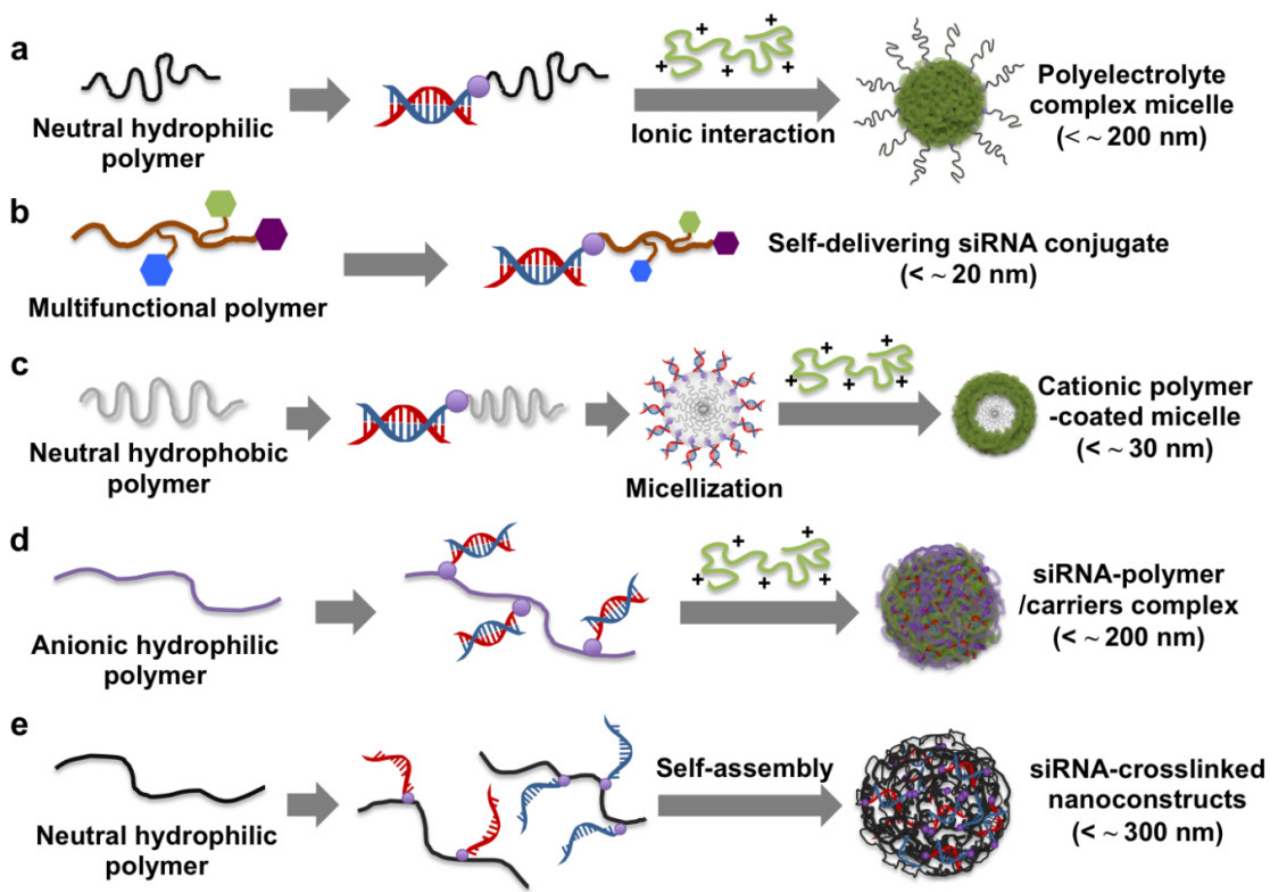

Figure 2. Schematic illustration of the preparation and delivery strategies of different siRNA-polymer conjugates.

\subsection{Hydrophobic polymers conjugated siRNA delivery systems}

Biodegradable solid polymers have also been used in a siRNA-polymer conjugation approach. Poly(lactic-co-glycolic acid) (PLGA) is a biodegrada- ble and biocompatible polymer which has been utilized for conjugation to various molecules, such as small molecular drugs, proteins, antisense oligonucleotides, and siRNA. ${ }^{41-44}$ An amphiphatic structure of an A-B type block copolymer was fabricated using siRNA-PLGA conjugates linked with disulfide bonds 
(Figure 2c). ${ }^{45}$ In an aqueous solution, the hydrophobic PLGA block was self-aggregated into an inner core, while the hydrophilic siRNA block formed an outer shell, generating spherical core/shell type micelles. The prepared siRNA-PLGA micelles had a diameter of about $20 \mathrm{~nm}$ and a zeta potential of $-16.6 \pm 2.6 \mathrm{mV}$. In particular, the clustered siRNA shell layer had a high spatial charge density, which enabled efficient interactions with cationic polyelectrolytes (e.g., linear polyethylenimine (LPEI) with a molecular weight (MW) of 2.5 and $25 \mathrm{kDa}$ ) to form cationic micelle structures. The LPEI-coated siRNA-PLGA micelles exhibited a greater extent of cellular uptake and gene silencing compared to the siRNA/LPEI complexes.

\section{4. siRNA-grafted polymer delivery system}

Recently, siRNA-grafted polymers have been proposed as a promising strategy for siRNA delivery. Hyaluronic acid (HA) is an anionic and natural polymer, which showed a strong affinity to CD 44 receptors on the cell membrane. Park et al. developed reducible siRNA-grafted HA conjugates for a target specific systemic delivery of siRNA to the liver (Figure $2 \mathrm{~d}) .{ }^{46}$ The conjugation of anionic HA (100 kDa) to siRNA enhanced a spatial charge density of siRNA, making it possible to form a tight complex with a weak polycation of LPEI with a molecular weight of $25 \mathrm{kDa}$. In vivo gene silencing efficiency of the MDA-MB-231 cells treated by the siRNA-HA/LPEI complexes was about $60 \%$, while naked siRNA/LPEI complexes showed no significant gene silencing.

siRNA-grafted dextran conjugates linked with disulfide bonds was also developed to fabricate dextran-coated siRNA complexes with LPEI $(25 \mathrm{kDa})$ for systemic siRNA delivery. ${ }^{47}$ Non-ionic, biocompatible, and anti-fouling dextran shells improved the structural stability of siRNA complexes in the presence of serum and showed tumor-targeting effects by the conjugation of folic acids to the dextran. In vivo study showed that folic acid-decorated dextran-grafted siRNA/LPEI complexes were efficiently transfected to target cells and induced sequence-specific gene silencing via a tail-vein injection to mice. In another study, two complementary single-stranded sense/ antisense siRNA-grafted dextran conjugates were molecularly hybridized to form siRNA-cross-linked nanoconstructs (NCs) (Figure 2e). ${ }^{48}$ TEM analysis revealed that the NCs had an average diameter of 100 $300 \mathrm{~nm}$ and a porous spherical structure. Notably, the NCs did not require cationic reagents for siRNA condensation, which reduced cytotoxicity and non-specific binding. The surface of NCs were coupled with the prostate-carcinoma-binding peptide aptamer, DUP-1, which showed target-specific siRNA delivery and gene silencing as compared to the NCs without the aptamer. More recently, we reported a new strategy for stable, compact nanogels incorporating siRNA via electrostatic interaction and chemical cross-linking between siRNA and LPEI (2.5 kDa) (Figure 3). ${ }^{49}$ Thiol-terminated siRNA at both $3^{\prime}$ ends were cross-linked with thiol-grafted LPEI via disulfide bonds, which allowed siRNA/LPEI complexes to be effectively condensed via inter- and intra-molecular networks. However, these mutually cross-linked siRNA/LPEI nanogels were readily dissociated in the cytoplasm, releasing free monomeric siRNA that can effectively trigger RNAi effects. In particular, the cross-linked LPEI also degraded into oligomeric LPEI, which was known to be less toxic than high-molecular-weight polyelectrolytes. The siRNA/LPEI nanogels showed a much higher level of cellular uptake in human epithelial carcinoma cells (HeLa cells) and suppressed about $80 \%$ of VEGF expression without cytotoxicity as compared to untreated cells. On the other hand, the siRNA/LPEI complexes with only LPEI-mediated cross-links showed no enhanced gene silencing and cellular uptake.

\section{3. siRNA-Loaded Encapsulated Lipo- somes}

Lipids can be spontaneously assembled to form liposomes with spherical lipid bilayers in an aqueous environment. ${ }^{50}$ A great variety of hydrophilic molecules, such as small drugs, proteins, and nucleic acids, can be entrapped within the hollow aqueous core during the formation of liposomes. ${ }^{51,52}$ The encapsulation of siRNA into liposomes can avoid nonspecific binding and degradation by proteins and enzymes in the bloodstream, increasing the circulation time of siRNA. In addition, the outer surface of liposomes can be modified with various moieties through a post-insertion method and by means of directed conjugation to the lipids. In particular, the lipid bilayers of liposomes can be fused with other lipid bilayers, facilitating the internalization of liposomes into the cell membrane. These types of siRNA-encapsulated liposomes have been proposed as a promising vehicle for stable, efficient systemic delivery. A typical structure of liposomes encapsulating siRNA is shown in Figure 4. The representative examples of siRNA-loaded encapsulated liposomes for siRNA delivery are listed in Table 2 with the diameter and zeta potential of nanoparticles, target genes, and target cells. 
$\mathbf{a}$
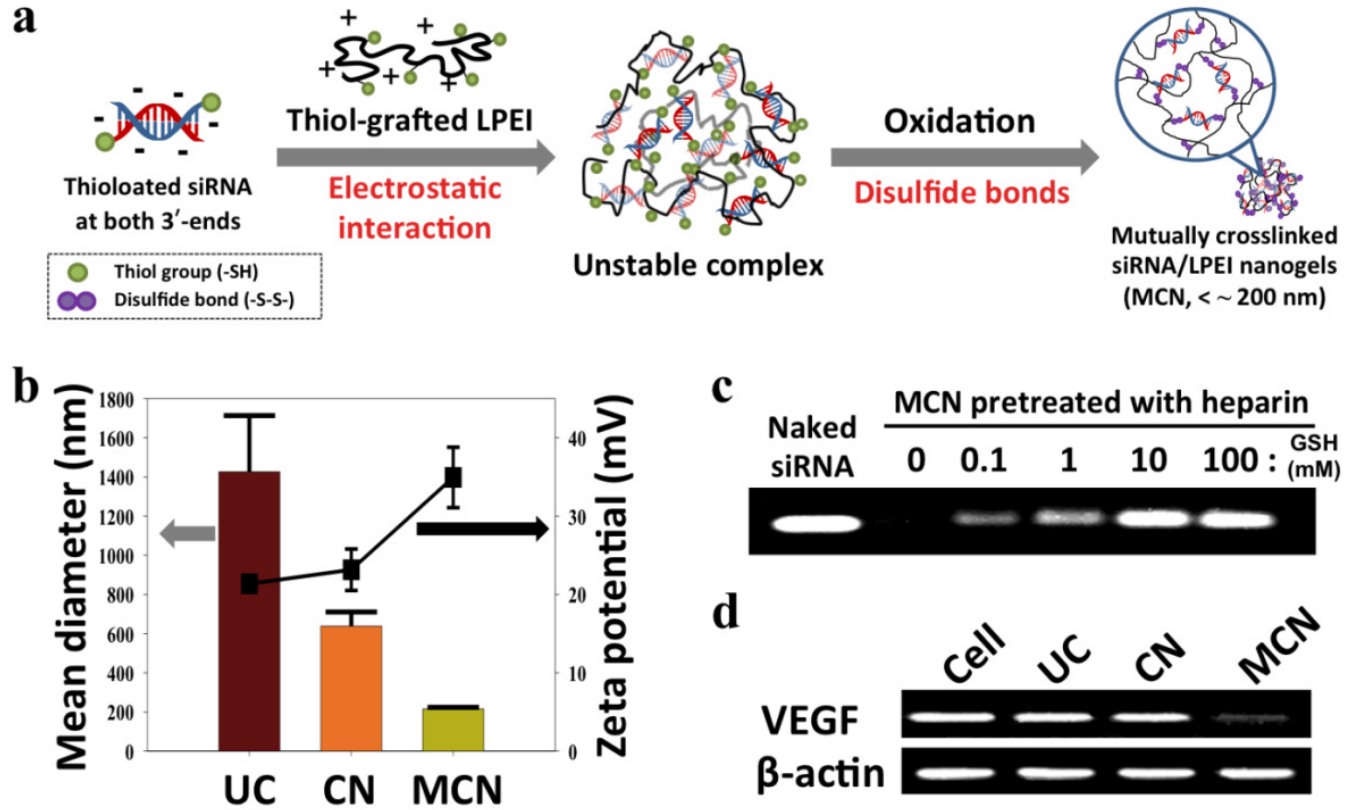

C

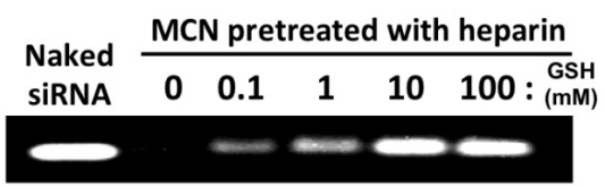

d

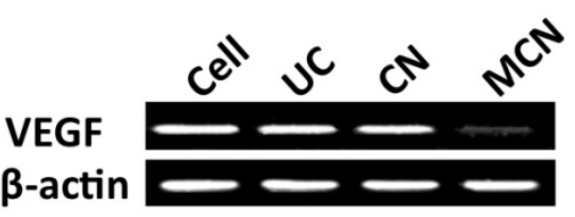

Figure 3. (a) Synthetic scheme of the construction of reductively dissociable siRNA-polymer nanogels (MCN), (b) hydrodynamic mean diameters (bar graph, left panel) and zeta potentials (square, right panel) of different siRNA complexes, (c) visualization of released siRNA from nanogels in a heparin solution while increasing the glutathione (GSH) concentration, and (d) semi-quantitative RT-PCR analysis of intracellular VEGF mRNA in cells after the transfection of different siRNA complexes. Human $\beta$-actin mRNA was used as a control. UC denotes siRNA/LPEI complexes, CN represents siRNA/thiol-grafted LPEI complexes, and MCN denotes thiolated siRNA/thiol-grafted LPEI complexes. Copyright 2012 Wiley-VCH.

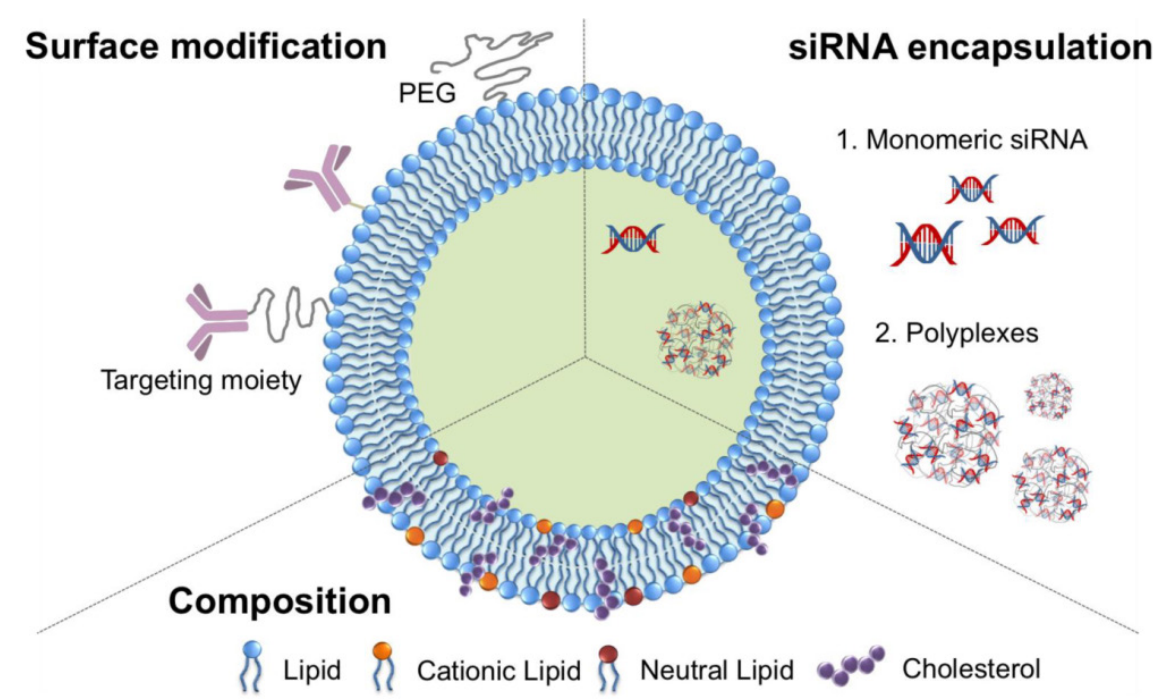

Figure 4. A schematic design of liposomes encapsulating siRNA.

Table 2. Summary of siRNA-loaded encapsulated liposomes used in siRNA delivery.

\begin{tabular}{|c|c|c|c|c|c|}
\hline Liposome components & Diameter (nm) & Zeta potential $(\mathrm{mV})$ & Target genes & Target cells & Ref. \\
\hline Egg PC, Ch, PEG-PE, DOTAP, Arginine octamer (R8) & 150 & $+5.5 \pm 1.7$ & HDM2 & SK-MES-1 & 53 \\
\hline DOTAP, Ch, PEG-lipids & $189.5 \pm 3.7$ & $+49.8 \pm 4.0$ & GFP & HeLa & 54 \\
\hline DOPC & Not shown & Not shown & EphA2 & HeyA8, SKOV3ip1 & 55 \\
\hline DOTAP, DOPE, PEG-PE, Ch, Anti-EGFR/Arginine 12 (R12) & 200 & Not shown & Luciferase & NCI-H322 & 56 \\
\hline DOTAP, Ch, Anisamide-DSPE-PEG, DSPE-PEG, & 120 & +45 & Luciferase & B16F10 & 57 \\
\hline DLinDMA, DSPC, Ch, PEG-C-DMA & $141 \pm 14$ & $+20.2 \pm 7.9$ & HBV263, HBV1583 & Hep2 & 60,61 \\
\hline Lipidoid, Ch, PEG-lipids & $50 \sim 80$ & $+2 \sim 34$ & Factor VII, ApoB & Hepatocytes & $62 \sim 64$ \\
\hline
\end{tabular}

PC, phosphatidylcholine; Ch, cholesterol; DOTAP, 1,2-dioleoyl-3-trimethylammonium-propane; DPPE, 1,2-dipalmitoyl-sn-glycero-3-phosphoethanolamine; DOPC,

1,2-dioleoyl-sn-glycero-3-phosphatidylcholine; DSPE-PEG, distearoyl-sn-glycero-3-phosphoethanolamine-n-[methoxy (polyethyleneglycol)]; DOPE,

1,2-dioleoyl-sn-glycero-3-phosphoethanolamine; PEG-PE, polyethylene glycol-1,2-distearoyl-sn-glycero-3-phosphatidylethanolamine; PEG-C-DMA, 3-N-[ $\omega$-Methoxy poly(ethylene glycol)carbamoyll]-1,2-dimyristyloxypropylamine; DSPC, 1,2-Distearoyl-sn-glycero-3-phosphocholine; DLinDMA,

1,2-dilinoleyloxy-N,N-dimethyl-3-aminopropane. HDM2, mouse double minute 2 homolog; EphA2, ephrin type-A receptor 2; HBV, hepatitis B virus; ApoB, apolipoprotein 


\section{I. Encapsulation of siRNA in liposomes via the rehydration of dried lipid films}

The simplest method to encapsulate siRNA into the core of liposomes is to mix a dry, thin lipid film with an aqueous siRNA solution. For example, a dry lipid film composed of phosphatidylcholine, cholesterols, cationic lipids, and PEG-lipids was hydrated with a siRNA solution to form siRNA-encapsulated cationic liposomes. ${ }^{53}$ The cationic lipids (e.g., 1,2-dioleoyl-3-trimethylammonium-propane

(DOTAP)) were electrostatically complexed with siRNA, allowing the efficient encapsulation of siRNA into liposomes. The outer surfaces of the cationic liposomes were conjugated with arginine octamers (R8) as cell-penetrating peptides for enhanced cellular uptake and gene silencing. The resultant R8-liposomes encapsulating siRNA effectively inhibited the targeted gene and significantly reduced the proliferation of cancer cells.

\subsection{Encapsulation of siRNA in liposomes via the dehydration-rehydration process}

Several studies have described the application of lyophilization for the formulation of siRNA-loaded liposomes. This process maintains the biological activity of siRNA and the structural stability of the lipid bilayers. The addition of lyoprotectants such as glucose, sucrose, trehalose and lactose can also contribute to the stabilization of liposomes during the drying process, producing stable and dispersed liposomes. PEGylated cationic liposomes were employed to encapsulate siRNA via hydration with a freeze-dried matrix (HFDM) method. ${ }^{54}$ Briefly, siRNA in a sucrose solution was mixed with a mixture of lipids of DOTAP, cholesterol, and PEG-lipids, in butanol. The mixture was freeze-dried and rehydrated to form siRNA-loaded liposomes. The prepared PEGylated liposomes had diameters of $189.5 \pm 3.7 \mathrm{~nm}$ and a zeta potential of $49.8 \pm 4.0 \mathrm{mV}$. There was no significant change in these values for 1 month at $4{ }^{\circ} \mathrm{C}$ and at room temperature. The level of GFP expression was decreased by $80 \%$ in HeLa cells by transfection with PEGylated cationic liposomes.

Despite the enhanced cellular uptake and encapsulation of siRNA, cationic liposomes can activate an immune response in vivo. To solve this problem, neutral liposomes were employed to incorporate siRNA. 55 Neutral 1,2-oleoyl-sn-glycero-3-phosphocholine (DOPC) was mixed with siRNA in the presence of excess butanol and then lyophilized. This process exhibited siRNA encapsulation efficiency of about $65 \%$ in the core of the liposomes, which was relatively low compared to that by cationic liposomes (about 90 \%). These neutral liposomes encapsulating
siRNA showed a significant decrease of EphA2 expression in vitro and in vivo. In addition, liposomes co-encapsulating siRNA and paclitaxel also dramatically suppressed tumor growth via an i.v. injection. The surface charge of the liposomes has been shown to affect the in vivo distribution and location of liposomes. Very interestingly, neutral liposomes showed more enhanced accumulation in tumor tissues compared with cationic DOTAP-based liposomes. This result indicated that the surface charge of liposomes is an important factor in determining the cellular uptake and gene silencing efficiency.

Recent research has shown that two types of lipid-inverted micelles can be mixed and evaporated to form asymmetric liposome particles (ALPs) with different components in their inner and outer layers, ${ }^{56}$ The inner layer encapsulating siRNA was composed of cationic lipids, and the outer layer was composed of anionic lipids, PEG, and cholesterols. The ALPs achieved more than $95 \%$ siRNA encapsulation while also protecting the siRNA from ribonuclease A and other serum proteins. However, the ALPs were not efficiently internalized into cells without surface modification with a polyarginine peptide (R12) or anti-human epidermal growth factor receptor antibody (anti-EGFR) due to their negative surface charge. The surface-modified ALPs showed enhanced cellular uptake and dose-dependent gene silencing. In particular, the anti-EGFR-ALPs induced the cell-specific delivery of siRNA via receptor-mediated endocytosis.

\subsection{Liposomes encapsulating with siRNA complexes}

To obtain a high loading efficiency, siRNA was pre-condensed with cationic polymers, peptides, and calcium phosphate and then incorporated into the core of liposomes. It was demonstrated that cationic polypeptide, protamine, and a mixture of siRNA and HA were mixed to form negatively charged complexes with about $150 \mathrm{~nm}$ in diameter. ${ }^{57}$ Cationic lipids were added to coat the siRNA/HA/protamine complexes via electrostatic interaction, thereby forming liposome-protamine-HA nanoparticles (LPH-NPs). The LPH-NPs were further modified with anisamide-PEG-lipids as a targeting ligand on their surface via a post-insertion method. The targeted LPH-NPs had a diameter of about $115 \mathrm{~nm}$, a zeta potential of $25 \mathrm{mV}$, and a loading efficiency of $90 \%$. An in vivo study showed that the LPH-NPs silenced $80 \%$ of luciferase activity in a metastatic B16F10 tumor in the lung after an i.v. injection $(0.15 \mathrm{mg}$ siRNA $/ \mathrm{kg})$. In a follow-up study, protamine was replaced with a histone-based recombinant protein, tetra-H2A (TH). ${ }^{58}$ The incorporated $\mathrm{TH}$ with cathepsin D cleavage sites 
was readily degraded to dissociate siRNAs from siRNA/HA/TH complexes due to the endosomal/lysosomal proteases, enhancing the cytoplasmic release of siRNA. The liposomes encapsulating the siRNA/HA/TH complexes exhibited a higher silencing efficiency of target genes in vitro and in vivo than the LPH-NPs. In a similar approach, siRNA was entrapped by rehydrating lyophilized neutral liposomes with water containing siRNA/protamine complexes, allowing about $80 \%$ entrapment efficacy of siRNA. ${ }^{59}$ HA was covalently attached to the surface of the liposomes, stabilizing the liposomes in vivo as well as serving as a point of attachment for a targeting ligand, the $\beta_{7}$ integrin monoclonal antibody.

The unstable complexes of siRNA with cationic polymers are easily dissociated in the bloodstream due to anionic biomolecules. The application of a liposomal coating around the siRNA complexes is a useful strategy to increase siRNA protections as well as drug loading efficiency of liposomes.

\subsection{Clinical trials of liposomes for RNAi therapeutics.}

Stable nucleic acid lipid particles (SNALPs) have been considered as one of the most promising lipid-based vehicles for siRNA therapeutics.60,61 SNALPs have a hydrodynamic diameter of $140 \mathrm{~nm}$ and an encapsulation efficiency of about $94 \%$ for siRNA. They are made of a mixture of lipids, including cholesterols and cationic and neutral lipids, which facilitate the cellular uptake and endosomal release of siRNA via lipid bilayer fusion. The outer surface of the SNALPs is also coated with PEG-lipid conjugates, a

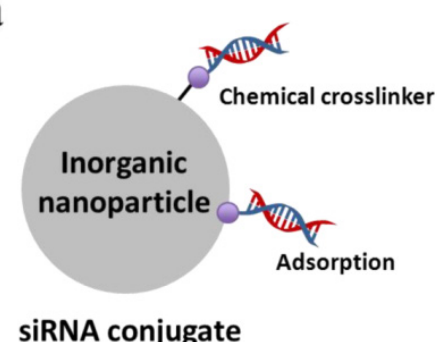

c

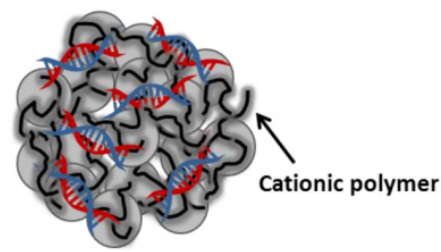

Nanoparticle cluster b

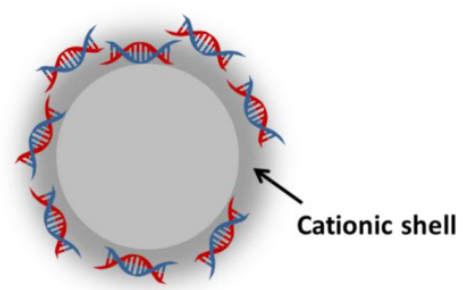

Electrostatic interaction

d

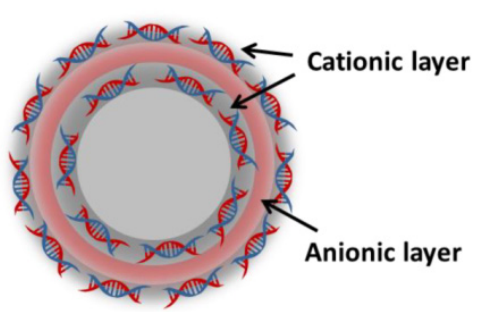

Layer-by-layer assembly which provide anti-fouling effects and structural stability. SNALPs exhibit efficient delivery to the liver and the knockdown of therapeutically relevant target genes, the hepatitis $B$ virus in the mouse and the lipoprotein ApoB in nonhuman primates, without cytotoxicity and an immunoresponse. Recently, the phase 1 clinical trial was completed for SNALPs encapsulating ApoB-specific siRNA. In another groups, the synthesis, characterization, and identification of the optimal siRNA carriers with lipid-like molecules termed as 'lipidoids' were investigated. ${ }^{62-64}$ The lipidoids were composed of the amine-rich central part attached to long oxoalkyl chains. The lipidoids nanoparticles encapsulated with siRNAs showed a diameter of $50 \sim 80 \mathrm{~nm}$, a zeta potential of $+2 \sim 34 \mathrm{mV}$, and siRNA encapsulation efficiency of $90 \sim 95 \%$. Among the huge of lipidoids, $98 \mathrm{~N}_{12}-5$ lipidoid-based siRNA nanoparticles inhibited $75 \sim 90 \%$ of Factor VII or ApoB expression in hepatocytes in mice or nonhuman primates at $5 \mathrm{mg} / \mathrm{kg}$ doses. Especially, C12-200 lipidoids showed the best-performing gene silencing, inhibiting $>80 \%$ of Factor VII expression at $0.03 \mathrm{mg} / \mathrm{kg}$ of siRNA into mice. Notably, five different hepatic genes $(0.1 \mathrm{mg} / \mathrm{kg}$ total siRNA dose) were simultaneously silenced by a single i.v. injection of C12-200 lipidoids encapsulating pooled siRNAs.

\section{Inorganic Nanoparticle-based siRNA Delivery Systems}

Recent advances in the synthesis process and surface modification of inorganic nanoparticles have led to the development of siRNA delivery and molecular imaging techniques. The functionalized surfaces of inorganic nanoparticles can easily be immobilized with various moieties, such as therapeutic drugs, targeting ligands, fluorescent dyes, nucleic acids and polymers. In addition, the unique optical and electronic properties of inorganic nanoparticles make it useful as a nanoprobe for biomedical diagnosis and analysis. Several strategies to immobilize siRNA onto the surface of inorganic nanoparticles are highlighted in Figure 5. Here, we describe representative inorganic nanoparticles, such as gold nanoparticles (AuNPs), magnetic nanoparticles, and quantum dots (QDs), for siRNA delivery.

Figure 5. Strategy to load siRNA on inorganic nanoparticles: (a) Chemical conjugation and adsorption of siRNA on a single nanoparticle surface; (b) electrostatic interaction of siRNA and cationic shell on (b) a single nanoparticle and (c) cationic polymer-coated nanoparticle clusters; (d) layer-by-layer assembly of siRNA and cationic polymers on a single nanoparticle surface. 


\section{I. Gold Nanoparticles}

AuNPs have been widely utilized as a gene delivery vehicle due to their biocompatibility, efficient translocation, size diversity, and surface functionalization. ${ }^{65}$ Their unique surfaces have provided the efficient attachment of siRNA onto AuNPs via chemisorption and electrostatic interaction. However, there are critical issues such as immune responses and long-term cytotoxicity for AuNP-based applications. Further studies seem to be necessary to clarify how physical and chemical characteristics of AuNPs affect their biological responses.

\section{I.I. Adsorption and chemical conjugation of siRNA on the AuNP surfaces}

The first application of AuNPs to siRNA delivery was reported in 2006.66 An siRNA bearing a thiol group at the $5^{\prime}$ end of the sense strand and PEG-poly[2-(N, N-dimethylamino)ethyl methacrylate] copolymer (PEG-PAMA) were co-decorated on the surfaces of AuNPs with diameters of $15 \mathrm{~nm}$ via Au-S interactions. Approximately 45 siRNA molecules were attached onto each AuNP. It was interesting to note that the AuNPs co-loaded with siRNA and PEG could penetrate the cell membrane without transfection agents, showing significant inhibition of luciferase expression in HuH-7 cells. In another study, the surface of AuNPs was modified with PEG, after which siRNA was conjugated to the terminal end of the PEG chain via a disulfide cross-linker.67 The loading efficiency of siRNA per AuNP increased with an increase in the salt concentration due to the reduction of electrostatic repulsion, resulting in that about 30 siRNA molecules were loaded onto each AuNP with a diameter of $15 \mathrm{~nm}$. The multiple siRNAs on the AuNP surface allowed an effective coating with a biodegradable cationic polymer, poly( $\beta$-amino ester)s (PBAEs) via electrostatic interactions. The PBAE-coated AuNPs reduced luciferase expression in HeLa cells up to $90 \%$, while no silencing effect was observed without the polymer coating. Giljohann et al. reported highly dense RNA monolayer-coated AuNPs termed as a polyvalent siRNA-AuNP conjugate. ${ }^{68}$ The sense siRNA-PEG-thiol conjugates were absorbed onto the AuNPs and hybridized with antisense siRNA, after which added with PEG-thiol for enhanced structural stability. This approach enabled $33 \pm 4$ molecules of siRNA to be loaded onto each AuNP with a diameter of $13 \mathrm{~nm}$. These nanoparticles were efficiently transported into the cell without the use of a transfection agent, showing prolonged RNAi activity in vitro.

\section{I.2. Electrostatic interaction of siRNA on the cati- onic AuNP surfaces}

The ionic interactions between siRNA and AuNPs can also be used to pack siRNA onto AuNPs. For instance, amine-functionalized AuNPs were employed as a vehicle for siRNA delivery. ${ }^{69}$ To prevent uncontrollable ionic aggregation, a PEG chain was linked to the end of siRNA via disulfide bonds. The resultant siRNA-PEG conjugates electrostatically interacted with AuNP to form well-dispersed AuNP/siRNA-PEG complexes, resulting in a core-shell structure composed of a siRNA core and a PEG shell. Confocal laser scanning microscopy revealed that the AuNP/siRNA-PEG complexes were efficiently transported into the cell, resulting in enhanced gene silencing. In another study, triethylenetetramine (TETA)-terminated dendron was conjugated to the AuNP surface, forming dendronized AuNPs for siRNA delivery. ${ }^{70}$ AuNPs capped with G2 dendrons resulted in the most effective complexation with siRNA, leading to the suppression of the $\beta$-gal expression by about $50 \%$.

Recently, the surface modification of AuNPs can be easily prepared using cationic polymers as reducing and stabilizing agents. Song et al. reported that bPEI $(25 \mathrm{kDa})$ and $\mathrm{HAuCl}_{4}$ were mixed together in aqueous solution to produce bPEI-coated AuNPs for siRNA delivery. ${ }^{71}$ The resultant AuNP-PEI had a diameter of $18.2 \mathrm{~nm}$ and a zeta potential of $17.1 \pm 1.0$ $\mathrm{mV}$. Importantly, the charge density and steric effect of the coated bPEI maintained the original dispersion stability level and allowed the efficient deposition of siRNA through ionic interaction. The AuNP-bPEI/siRNA complexes induced the efficient gene silencing of exogenous GFP and endogenous polo-like kinase 1 (PLk 1) in MDA-MB-435-GFP cells. In a similar approach, catechol-grafted-bPEI (C-bPEI, $25 \mathrm{kDa}$ ) was used as a reducing agent as well as a cross-linker between the AuNPs, forming spherical bPEI-coated AuNP clusters. ${ }^{72}$ Their size and surface charge were controlled readily by adjusting the degree of catechol substitution in C-bPEI. The siRNA/bPEI-C-AuNPs were further modified with PEG to improve the dispersion stability in the presence of serum. The gene-silencing efficiency of the PEGylated siRNA/bPEI-C-AuNPs increased with an increase in the number of the catechol groups per bPEI.

\section{I.3. A layer-by-layer approach of siRNA and poly- mers on the AuNP surfaces}

To achieve a high loading efficiency of siRNA, a layer-by-layer (LBL) strategy was employed to form multiple shells on AuNPs by repeating oppositely charged polyelectrolytes. ${ }^{73}$ In addition, LBL has been used to tune the overall surface charge and to protect 
siRNA within the shielding layer from an enzymatic attack. Anionic AuNPs were electrostatically complexed with bPEI $(25 \mathrm{kDa})$ and then sequentially deposited siRNA and bPEI to form AuNP/bPEI/siRNA/bPEI complexes, which showed an increased hydrodynamic diameter of $22 \sim 25 \mathrm{~nm}$ compared to unmodified AuNPs with a diameter of $16 \mathrm{~nm}$. On average, about 780 siRNA molecules were complexed with each AuNP. The gene silencing effect was observed in CHO-K1 cells treated with AuNP/bPEI/siRNA/bPEI complexes compared to that of AuNP/bPEI/siRNA complexes. Some functional polyelectrolytes were also incorporated into the LBL for enhanced gene silencing. Charge-shifting AuNPs under an acidic environment were developed as a carrier for siRNA delivery. ${ }^{74}$ The surface of AuNPs were initially modified with negatively charged 11-mercaptoundecanoic acid (MUA) via Au-S bonding, after which bPEI (25 kDa) was deposited onto AuNP-MUA, forming cationic AuNP-MUA/bPEI as a core for LBL assembly. The cis-aconitic anhydride-functionalized poly(allylamine) (PAH-Cit) and bPEI (25 kDa) were sequentially deposited onto the AuNP-MUA/bPEI core via electrostatic interaction. The incorporated $\mathrm{PAH}-\mathrm{Cit}$ polyanions underwent a charge-reversal reaction, improving the endosomal escape of the nanoparticles due to an increased proton sponge effect. Confocal laser scanning microscopic images indicated that the charge-shifting AuNPs were widely distributed in the cytoplasm compared to nanoparticles formulated without PAH-Cit. The charge-shifting AuNPs (80 \%) showed more efficient gene silencing for lamin A/C protein expression than non-charge shifting AuNPs (20\%) and Lipofectamine 2000 (66\%). Recently, the same group reported that AuNPs were directly reduced and stabilized by biocompatible chitosan (CS) rather than by the conjugation of MUA to AuNPs, resulting in the substitution AuNP-CS for AuNPMUA/bPEI as a core for LBL assembly (Figure 6). ${ }^{75}$ The AuNP-CS/PAH-Cit/bPEI nanoparticles had a narrower size distribution with no reduction of the intracellular delivery efficiency of siRNA. They also induced significant suppression of MDR1 genes encoding $\mathrm{P}$ glycoprotein, a drug exporter membrane protein, allowing increased cellular uptake of doxorubicin in drug-resistant MCF-7 cells. Lee et al. also incorporated protease-degradable peptides, PLL, and siRNA on the surface of AuNPs to fabricate siRNA/PLL multilayer-coated AuNPs. ${ }^{76}$ The PLL multilayer was degraded by a cathepsin B enzyme in the cell to release the incorporated siRNA gradually, inducing a prolonged gene-silencing effect. Importantly, the relative level of luciferase suppression increasingly enhanced with the increased number of the siRNA layers.
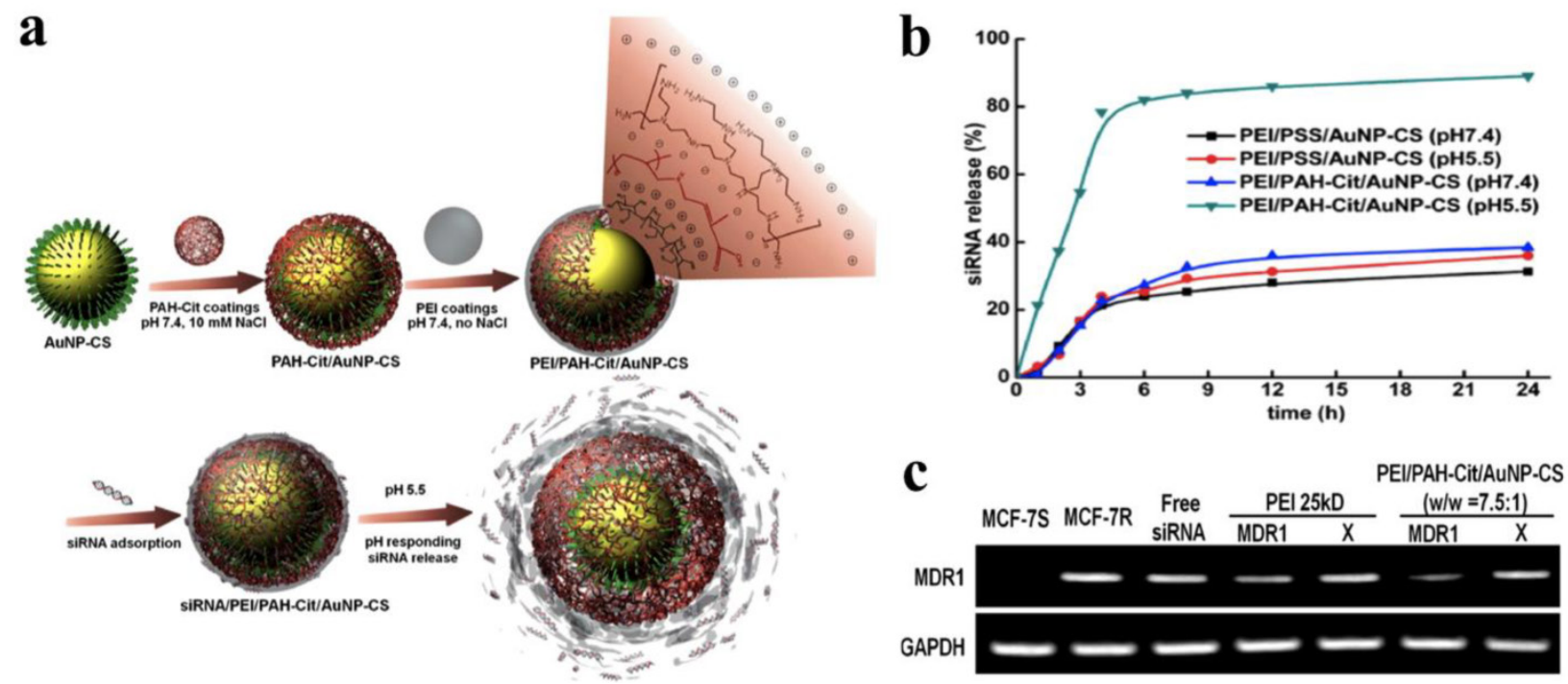

Figure 6. (a) Schematic illustrations of the construction of a siRNA/PEI/PAH-Cit/CS-AuNP nanoparticle and the pH-responsive release of siRNA, (b) quantitative analysis of a released siRNA from siRNA/PEI/PAH-Cit/CS-AuNP nanoparticle in a pH environment ranging from 7.4 to 5.5 . (c) MDRI mRNA levels in cells treated with bPEI (25 kDa)/siRNA complexes and siRNA/PEI/PAH-Cit/CS-AuNP nanoparticles. $\mathrm{X}$ is scrambled siRNA and GAPDH mRNA was used as a control. Copyright 2012 American Chemical Society.

\section{I.4. AuNP clusters for siRNA delivery systems}

Recently, HA was introduced to the outer layer of AuNP/siRNA/bPEI clusters for target-specific systemic delivery. ${ }^{77}$ Briefly, amine-functionalized AuNPs with a diameter of $13 \mathrm{~nm}$ were electrostati- cally complexed with siRNA to produce AuNP/siRNA clusters, after which they were sequentially coated with bPEI ( $25 \mathrm{kDa})$ and HA via LBL. The resultant clusters had a hydrodynamic diameter of $165.5 \pm 9.9 \mathrm{~nm}$ with a low polydispersity index and a zeta potential of $-12 \mathrm{mV}$. The anionic HA layer acted 
as a protein-resistant anti-fouling layer that prevents nonspecific interaction with serum proteins as well as target-specific delivery via HA receptor-mediated endocytosis. The AuNP/siRNA/bPEI/HA nanoparticles showed an excellent target-specific gene-silencing efficiency of $70 \%$ for B16F1 cells with HA receptors in the presence of $50 \%$ serum. Furthermore, they significantly reduced the mRNA level of apolipoprotein B to $20 \%$ in the liver following tail-vein injections to mice.

\section{I.5. AuNP-based photo-inducible RNAi therapeutics}

The surface plasmon resonance (SPR) absorption of AuNPs at near-infrared (NIR) laser has drawn increasing attention for applications to photo-inducible RNAi. The absorbed NIR light was inverted to heat energy, which increased the surface temperature of AuNP, resulting in the disintegration of Au-S bonds. In addition, NIR light $(700 \sim 900 \mathrm{~nm})$ can penetrate deep into the tissue without causing severe damage. The laser-dependent desorption of siRNA was reported for temporally and spatially controlled gene silencing. ${ }^{78}$ Thiolated siRNA bearing PEG at the $3^{\prime}$ end of the sense strand and Cy3 at the 5 ' end of the antisense strand were immobilized on the AuNP surfaces. The prepared AuNP-siRNA-Cy3 was capped with cationic trans-activator of transcription (Tat) peptide lipids via electrostatic interaction for improved cellular uptake and siRNA protection, forming a 3-nm-thick lipid bilayer on the AuNP having a diameter of about $40 \mathrm{~nm}$. Confocal microscopy analysis showed that multiple bright Cy3 fluorescent dots were present in the cytoplasm following the exposure of the transfected cells to a NIR laser. The GFP expression of C166-GFP cells treated with AuNP-siRNA was reduced to $80 \%$ by laser induction. Target-cell-specific gene silencing in vitro and in vivo via NIR-induced release of siRNA from AuNPs was also reported. ${ }^{79}$ Folic acid (FA) was introduced as a tumor-targeting moiety onto the AuNP attached with siRNA via Au-S bonds. The fabricated FA-PEG-AuNP-siRNA nanoparticles were internalized into HeLa cells via receptor-mediated endocytosis, inducing a gene inhibition rate of $92 \%$ for $N F-\kappa B$ p65 after NIR irradiation compared to scrambled siRNA incorporated FA-PEG-AuNP-siRNA. In experimental tumor models, the effective down-regulation of the $N F-\kappa B p 65$ was achieved only in tumors stimulated with NIR irradiation following an i.v. injection of FA-PEG-AuNP-siRNA. However, organs without NIR exposure did not show any gene silencing activity despite intracellular translocation. Notably, NIR light exhibited the disruption of endocytic vesicular membrane due to the heating of the surrounding the AuNPs, facilitating endosomal re- lease and RNAi activity. Recently, an AuNP shell functionalized with PLL was used to capture and release siRNA molecules in response to NIR light. ${ }^{80}$ The light-triggered gene silencing was demonstrated to decrease the expression of GFP to $49 \%$ in targeted cells, while treatment without laser had only $80 \%$.

\subsection{Magnetic Nanoparticles}

Magnetic nanoparticles have been widely used as a magnetic resonance imaging (MRI) contrast agents for medical diagnoses of the liver, spleen, and bone marrow. ${ }^{81}$ Moreover, recent studies have attempted to apply magnetic nanoparticle-based gene delivery systems for simultaneous diagnostic and therapeutic functions in vivo. 82,83 For effective gene delivery, it is necessary to modify the surface of magnetic nanoparticles for the stable attachment of therapeutic molecules. This surface functionalization process can allow the effective loading of siRNA onto magnetic nanoparticles.

\subsection{Chemical conjugation of siRNA on magnetic nanoparticles}

Efficient siRNA delivery was reported using two-layered iron oxide nanoparticles (IONPs, $8 \mathrm{~nm}$ in diameter) stabilized with two different polymers. ${ }^{84}$ The polymer-stabilized IONPs were structured by an inner layer of poly(oligoethylene glycol) methyl ether acrylate, poly(OEG-A), which can be complexed with siRNA, and an outer layer of poly(dimethylaminoethyl acrylate), poly(DMAEA), which has anti-fouling effects. Gene silencing in GFP-overexpressing cells after the incubation of the two-layered IONP/siRNA nanoparticles showed 50 $\%$ and $80 \%$ levels in the presence and absence of a magnetic field, respectively, compared to untreated cells.

\subsubsection{Magnetic nanoparticles for simultaneous siRNA delivery and molecular imaging}

Simultaneous imaging and delivery of siRNA into target cells has also been attempted using a magnetic nanoparticle. For example, the aminated dextran-coated magnetic nanoparticles were covalently linked with siRNA, Cy5.5 dyes, and membrane-translocating peptides, allowing for real-time monitoring of the treatment. ${ }^{85} \mathrm{~A}$ remarked drop in $T_{2}$ relaxation times and high fluorescence were found in the target tumors following systemic administration. In addition, their presence in tumor cells reduced the mRNA level of targeted genes down to $85 \pm 2 \%$, resulting in the optical imaging to corroborate the RNAi activity. Notably, these probes did not carry a tumor-targeting moiety; however, they were accumulated in tumor cells presumably due to enhanced 
permeability and retention induced by leaky vasculature in the tumor tissue compared to the tight endothelial junctions of normal blood vessels. ${ }^{86}$ In another study, dendrimer-conjugated magneto-fluorescent nanoworms, dendriworms, composed of a series of about 7 iron oxide nanoparticles were also suggested as carriers and imaging agents for in vivo siRNA delivery. ${ }^{87}$ To prepare dendriworms, amine-modified nanoworms were labeled with NIR dyes, and subsequently conjugated with cysteamine core poly(amino amine) (PAMAM) G4 dendrimers via a reducible cross-linker. The resultant dendriworms showed a zeta potential of $16 \sim 24 \mathrm{mV}$ and a diameter of $80 \sim 100 \mathrm{~nm}$. Dendriworms carrying siRNA remarkably suppressed GFP expression in human glioblastoma cells in vitro, which was 2.5-fold more efficient than commercial cationic lipids. In addition, they led to significant inhibition of GFP expression in a GFP-driven transgenic model of glioblastoma and simultaneously enabled fluorescent tracking of siRNA delivery following a direct i.v. injection into a mouse brain. More recently, a cell-specific hollow manganese oxide nanoparticle (HMON) probe, which generated a bright signal density on $T_{1}$-weighted imaging, was proposed. ${ }^{88}$ The surface of the probe was coated with 3,4-dihydroxy-L-phenylalanine (DOPA)-conjugated bPEI $(25 \mathrm{kDa})$ through catechol-metal coordination and then functionalized with a therapeutic monoclonal antibody, Herceptin, which specially binds to the human epidermal growth factor receptor 2 (HER2). Confocal microscopy and MRI imaging studies revealed that HMON-bPEI-Herceptin/siRNA nanoparticles specifically detected HER2-overexpressing cancer cells due to ligand-receptor interaction while also efficiently inhibiting VEGF expression.

\subsubsection{Target-cell-specific magnetic nanoparticles.}

To enhance intracellular translocation, cell-targeting RGD peptides were introduced onto the surfaces of magnetic nanoparticles functionalized with siRNA and Cy5 dyes. ${ }^{89}$ The surface-bound siRNA numbered about $40 \pm 1$ per nanoparticle, and the siRNA efficiently inhibited GFP expression. These probes showed a strong $T_{2}$-weighted contrast and bright red fluorescence in MDA-MB- 435 cells due to RGD-mediated endocytosis. In another group, the targeting peptide chlorotoxin (CTX) and siRNA were covalently attached onto the surface of magnetic nanoparticles surrounded with PEG-grafted chitosan and bPEI $(2 \mathrm{kDa}) .{ }^{90}$ The resultant nanoparticles had a hydrodynamic size of $111.9 \pm 52.4 \mathrm{~nm}$, a zeta potential of $19.6 \pm 9.7 \mathrm{mV}$, and a magnetic relaxation $\left(R_{2}\right)$ value of $214.07 \mathrm{~S}^{-1} \mathrm{mM}^{-1}$. Gel retardation assays indicated that about 3.8 siRNAs and 5 CTXs were immobilized onto each nanoparticle. GFP-overexpressing C6 cells treated with CTX-functionalized nanoparticles showed a strong $\mathrm{R}_{2}$ signal ( 4.2 fold) and significantly inhibited GFP expression (62\%) compared to untreated cells. In a follow-up study, three types of cationic polymers (polyarginine, PLL, and PEI) were used as a coating layer of magnetic nanoparticles and were evaluated in terms of their gene-silencing efficiency and intracellular pathway characteristics. ${ }^{91}$ Gene-silencing activity and cell viability assessments demonstrated that polyarginine-coated nanoparticles were most effective while also showing the lowest level of toxicity. Furthermore, TEM analysis revealed that polyarginine-coated nanoparticles entered cells through cell transcytosis, while PLL and PEI-coated nanoparticles were translocated into cells via endocytosis. These results indicated that the gene silencing and intracellular pathway characteristics depended on the coating materials on the surfaces of the nanoparticles. Recently, the same groups also developed $\mathrm{pH}$-sensitive magnetic nanoparticles. ${ }^{92}$ These nanoparticles were coated with primary amine group-blocked bPEI (25 kDa), CTX, and siRNA. The blocked bPEI was readily recovered to primary amine groups under an acidic environment, which facilitated cellular uptake. The resulting nanoparticles exhibited a significantly increased gene-silencing effect at $\mathrm{pH}$ 6.2 compared to that at $\mathrm{pH} 7.4$ as well as a strong $\mathrm{T}_{2}$-weighted signal in cells.

\subsubsection{Magnetofection-based siRNA delivery systems}

Magnetic nanoparticles have been introduced to concentrate the nanoparticles at a specific target cells through the application of an external magnetic field. This magnetic-guided drug delivery, termed as magnetofection, showed high transfection efficiency in hard-to-transfect cells such as primary cells and neuronal cells. It is conceivable that effective transfection can be achieved to increase the magnetic responsiveness. One approach is to assemble individual nanoparticles to a cluster. Namiki et al. developed a magnetic crystal-lipid nanostructure, called LipoMag, comprising an oleic acid-coated magnetic nanocrystal cluster in the core and a cationic lipid in the shell..$^{93}$ Compared to PolyMag, a commercial magnetic vehicle, LipoMag carrying siRNA exhibited more efficient gene transfection and gene silencing in a tumor tissue under a magnetic field. A tumor volume reduction of nearly $50 \%$ was noted in mice treated with LipoMag/siRNA nanoparticles upon a 28-day post i.v. injection, while no significant anti-tumor effect was observed in PolyMag/si siRNA-treated mice. It was also reported that oleic acid-coated magnetic nanocrystals were encapsulated in the core of polymeric nanocapsules via an oil-in-water emulsion method. ${ }^{94}$ An amphiphilic tri-block copolymer, Pluronic ${ }^{\circledR}$ F127, 
was dissolved in an organic solvent containing oleic acid-coated magnetic nanocrystals and the mixtures were then emulsified ultrasonically in an aqueous bPEI $(2 \mathrm{kDa})$ solution. The emulsion interface was stabilized by a cross-linking network between Pluronic ${ }^{\circledR}$ F127 and bPEI, encapsulating the magnetic nanocrystal clusters within the internal region. The fabricated nanocapsules were complexed with siRNA-PEG conjugates, which contributed to the steric stabilization of the polymeric nanocapsules. In the presence of an external magnetic field, the cellular uptake of the nanocapsules and subsequent gene silencing were effectively increased.

\subsubsection{Magnetic clusters for siRNA delivery systems}

DOPA-conjugated bPEI (25 kDa) can be employed as a cross-linker between $10 \mathrm{~nm}$ magnetic nanocrystals to form a spherical magnetic cluster with a diameter of $156.2 \pm 3.1 \mathrm{~nm}$ via oil-in-water emulsification. ${ }^{95}$ The surfaces of bPEI-coated magnetic clusters were further modified with PEG for steric stabilization and anti-fouling effects. The number of magnetic clusters taken by the cells was 6.6 times higher when they were exposed to an external magnetic field compared to that by unexposed cells. Alkylated bPEI (2 kDa) (alkyl-bPEI) was also employed to form a cluster structure of multiple magnetic nanocrystals. ${ }^{96}$ The alkyl-bPEI coated magnetic clusters had diameters of about $100 \mathrm{~nm}$ and a zeta potential of $40.8 \mathrm{mV}$. The siRNA-loaded alkyl-bPEI coated magnetic clusters enhanced the downregulation of luciferase in luciferase-overexpressing 4T1 cells (fLuc-4T1) and fLuc-4T1 tumors stably in a xenograft model after an intratumoral injection. In additional, the cells transfected by alkyl-bPEI-coated magnetic clusters displayed strong signal contrast compared to untreated cells according to $T_{2}$-weighted imaging due to a higher spin-spin relaxation time.

\subsection{Quantum Dots}

Quantum dots (QDs) are semiconductor nanocrystals that are generally composed of a semiconductor core (e.g., CdSe, InP and PbSe) enclosed in a shell layer of ZnS. ${ }^{97}$ Compared to organic dyes and fluorescent proteins, QDs exhibit outstanding optical properties such as size-tunable light emission, signal brightness, superior photostability, and broad adsorption. QD diameter sizes range from 2 to $10 \mathrm{~nm}$, allowing them to be excreted rapidly from the body via renal clearance. However, the surface oxidation and release of the toxic metal ions from QDs remain a significant challenge to those seeking to apply them biologically. ${ }^{98}$ One possible approach for solving this problem is a passivation shell with polymers, thus increasing the structural stability and biocompatibil- ity of QDs. In addition, the PEGylation of the QD surface has also offered the enhanced dispersion and immobilization of diverse biomolecules such as fluorescent dyes, targeting ligands, and therapeutic drugs.

\subsection{Chemical conjugation of siRNA on the QD surfaces}

An siRNA and tumor-homing peptides (F3 peptides) targeting cell surface were conjugated to the end of PEG on PEGylated QDs via chemical cross-linkers. ${ }^{99}$ The addition of endosome-disrupting agents facilitated the escape of the PEGylated QD-siRNA/F3 peptides nanocarriers from endosomes, resulting in an enhanced gene-silencing efficiency. In addition, it was found that the conjugation chemistry pertaining to the surfaces of QDs was a key factor affecting the gene-silencing efficiency. The reducible linkages between siRNA and QDs showed a greater gene-silencing effect than non-cleavable linkages. In another study, cell-penetrating HIV-Tat peptides were attached to the PEGylated QDs modified with therapeutic siRNA and cell-targeting RGD peptides for enhanced endosomal escape. ${ }^{100}$ They showed significant decreases in an epidermal growth factor receptor variant III (EGFRvIII) expression in target human U87 glioblastoma cells, triggering cell apoptosis. In addition, TEM images of the cells treated with the nanoparticles showed the presence of QDs in the cytoplasm. Yezehlyev et al. also reported the proton-sponge polymer coated QDs as a carrier and probes for siRNA delivery. ${ }^{101}$ These functions were achieved by converting carboxylic acid groups to tertiary amine groups on the QD surface. The resultant QDs not only allowed siRNA binding on the surface via electrostatic interaction but also facilitated the dissociation and release of siRNA into the cytoplasm. Two copies of siRNA were adsorbed onto the surface of a single QD with a diameter of $17 \mathrm{~nm}$. The QD/siRNA complexes exhibited a $10 \sim 20$-fold improvement in their gene-silencing efficiency and a $5 \sim$ 6 -fold reduction in the level of cytotoxicity in MDA-MB-231 cells compared to the commercial transfection agents Lipofectamine 2000, JetPEI, and TransIT.

\subsubsection{Cationic polymers-coated QDs for siRNA de- livery systems}

The surfaces of QDs modified with carboxylic acid groups were also employed to be cross-linked with the primary amine groups of chitosan via a 1-ethyl-3-(3-dimethylaminopropyl) carbodiimide (EDC) reaction, forming spherical and monodispersed chitosan-coated QD clusters with a diameter of about $60 \mathrm{~nm} .{ }^{102}$ The resultant chitosan nanoparticles encapsulating QDs were conjugated with siRNA and HER2 
ligand. This negative charge helped to facilitate the receptor-mediated endocytosis, resulting in efficient cell-specific delivery and gene silencing in SKBR3 breast cancer cells. In a similar approach, bPEI ( 25 $\mathrm{kDa}$ ) was used as a cross-linker between QDs to produce bPEI-coated QD clusters to monitor cellular processing and gene silencing of siRNA via fluorescence resonance energy transfer (FRET) signals. ${ }^{103}$ The FRET intensity in cells treated with QD-bPEI/Cy5-siRNA complexes dramatically decreased at $2 \mathrm{~h}$ after transfection, releasing Cy5-siRNA from the QD-bPEI/siRNA complexes. In addition, a protein transduction domain from human transcriptional factor Hph-1 conjugated QD-bPEI/Cy5-siRNA complexes facilitates cellular uptake and the unpacking kinetics of Cy5-siRNA in the cell. QD-bPEI/siRNA/HpH-1 showed much higher gene silencing efficiency than siRNA/bPEI complexes.

Maleic anhydride-alt-1-tetradecene was also used as an amphiphilic copolymer to link bPEI (25 $\mathrm{kDa}$ ) to the surface of QDs via EDC chemistry, forming bPEI-coated QDs with a diameter of $32.2 \mathrm{~nm} .{ }^{104} \mathrm{To}$ obtain cell-specific gene silencing, siRNA was conjugated to aptamers targeting a prostate-specific membrane antigen via a disulfide bond. Gel electrophoresis analysis indicated that about 20 copies of siRNA molecules were attached to each QD-bPEI. The QD-bPEI/siRNA-aptamer complexes showed a hydrodynamic diameter of $66.3 \pm 2.2 \mathrm{~nm}$ and a zeta potential of $-1.8 \pm 0.6 \mathrm{mV}$. FACS results showed that the GFP fluorescence intensity of C4-2B cells treated with the QD-bPEI/siRNA-aptamer complexes was significantly suppressed to $29.7 \%$ of the original value. However, the QD-bPEI/siRNA complexes without a targeting moiety did not significantly reduce the level of GFP expression.

\subsubsection{Functional molecules-coated QDs}

Amphipols are linear polymers with alternating hydrophilic and hydrophobic side chains. They are broadly used to solubilize hydrophobic membrane proteins into lipid bilayers. Interestingly, amphipol-coated QDs showed efficient cytosolic delivery, endosome escape capabilities, and siRNA protection during an enzymatic attack. ${ }^{105}$ Approximately 10 siRNA molecules were loaded per amphipol-QD. Compared to Lipofectamine 2000 and PEI ( $25 \mathrm{kDa})$, the QD-amphipol /siRNA complexes showed relatively high gene-silencing efficiency in the presence of serum without significant cytotoxicity. Time-lapse fluorescence imaging indicated that the QD-amphipol/FITC-labeled siRNA complexes penetrated the cell in $1 \mathrm{~h}$. The green fluorescence from FITC-siRNA started to appear in the cell in $1.5 \mathrm{~h}$ post-incubation and was homogeneously distributed in the cytoplasm at $5 \mathrm{~h}$, indicating efficient endosomal escape.

Cyclodextrin (CD) is a cup-shaped cyclic oligomer of glucose, which has an amphiphilic structure comprising a hydrophobic cavity and a hydrophilic exterior. ${ }^{106}$ Depending on the number of glucose units, they are classified as $\alpha, \beta$, and $\gamma$-CD for 6,7 , and 8 glucose units in a cyclic oligomer structure. ${ }^{107} \mathrm{CD}$ has been widely used as a drug delivery carrier due to its improved solubility in water, its high biocompatibility, and its low cytotoxicity. $\beta$-CD-grafted L-arginine-immobilized QD (QD- $\beta$-CD-L-Arg) was developed as a siRNA carrier and imaging probe. ${ }^{108}$ The guanidinium group of L-Arg shows a positive charge in a neutral environment, enabling the binding of siRNA to the surface of QD- $\beta$-CD-L-Arg. QD- $\beta$-CD-L-Arg/siRNA complexes exhibit higher gene-silencing efficiency than QD-L-Arg / siRNA complexes in terms of both mRNA and protein levels; in addition, both are better than commercial transfection agents. Notably, $\beta$-CD-coated QD-L-Arg carriers more efficiently protected siRNA from enzymatic degradation than QD-L-Arg carriers. $\beta$-CD molecules on the QD surface utilize steric hindrance to avoid siRNA degradation by ribonucleases. Recently, the same groups reported a co-delivery system composed of siRNA and a chemotherapeutic agent, doxorubicin (Dox), using QD- $\beta$-CD-L-Arg (or His). ${ }^{109}$ The Dox was initially loaded in the hydrophobic cavity of $\beta-C D$, and siRNA was then attached onto the QD-Dox- $\beta$-CD-L-Arg (or His). The QD-siRNA/Dox complex showed significant synergistic effects in a Dox-resistant cervical carcinoma cell line compared to QD-Dox complexes without siRNA.

Bacteriophage MS2 virus-like particles (MS2 VLP) based on QDs were developed to deliver therapeutic molecules such as chemotherapeutic drugs, siRNA, and protein toxins into human hepatocellular carcinomas for therapeutic and imaging agents. ${ }^{110}$ Briefly, MS2 coat proteins were self-assembled onto the pac site-modified QD surfaces to form 27.5 $\mathrm{nm}$-thick capsid layers. The layer can bind various cargos containing siRNA, pac site-conjugated doxorubicin, and the ricin toxin A-chain through a self-assembly process. The cargo-loaded MS2 VLP vehicles were further coupled with a targeting peptide, SP94, to improve cell-specific delivery, as well as a fusogenic peptide, H5WYG, to promote endosomal escape. The MS2 VLP carriers showed efficient and highly specific delivery of various drugs into target cells.

Recently, QD-incorporating solid lipid nanoparticles (SLNs) was developed for siRNA/drug combination therapy for cancers. ${ }^{111}$ The natural components of a low-density lipoprotein (LDL) were employed to 
form a stable core/shell nanostructure, LDL-mimetic SLNs, wherein cholesteryl oleate and triglyceride were embedded in an amphiphilic shell of phospholipids and cholesterols. The anticancer drugs, paclitaxel, and QDs ( $5 \mathrm{~nm}$ in diameter $)$ were co-encapsulated within the core of LDL-mimetic SLNs, while siRNA targeting against Bcl-2 were immobilized on their outer surface via electrostatic interactions. The nanoparticles showed the effective co-delivery of paclitaxel and siRNA into human lung carcinoma cells, leading to a significant synergistic effect in cancer therapy. In addition, strong fluorescence of the QDs enabled efficient monitoring of the intracellular translocation of SLNs into cancer cells.

\section{Engineered siRNA-based Structures with RNAi Activity}

Recently, uniquely designed siRNA motifs have been exploited as a building block to fabricate a variety of two- and three-dimensional architectures with RNAi activity via chemical/physical processes (Table 4). Compared to naked siRNA, engineered siRNA-based structures show spatially increased charge density, which dramatically facilitates the condensation of siRNA with low molecular cationic polymers to produce stable nanoparticles due to strong electrostatic interaction. ${ }^{112}$ In addition, the nanoparticles can readily degrade into free siRNA species loaded on RISC following cellular uptake. Such an approach has been recognized as a new strategy for highly efficient intracellular delivery of siRNA based on polymeric condensation. The first attempt of engineered siRNA-based gene silencing was reported by Bolcato-Bellemin et al. ${ }^{113}$ This groups developed sticky siRNA (ssiRNA) composed of short overhangs containing $A_{5-8} / T_{5-8}$ at both $3^{\prime}$ ends. The overhangs of ssiRNA have a low melting point of about $10{ }^{\circ} \mathrm{C}$. Interestingly, however, these overhangs enabled monomeric siRNA to be elongated to form siRNA oligomers upon complexation with JetPEI due to the reduced repulsive force, leading to the stable and compact nanoparticles. When compared to naked siRNA/Jet PEI complexes, these ssiRNA/Jet PEI complex exhibited increased RNase protection, structural stability in the presence of competing anionic polymers, and in vitro gene silencing up to 10-fold. Recently, Liu et al. also investigated the gene-silencing efficiency of ssiRNA while increasing the generation of triethanolamine (TEA)-core PAMAM dendrimers. ${ }^{114}$ The ssiRNA/dendrimers complexes showed significant gene silencing in vitro and in vivo with a fifth-generation dendrimer. However, naked siRNA required dendrimers of higher generations ( $>7$ ) for an RNAi effect.

In another study, chemical linkages were intro- duced to elongate monomeric siRNA to improve the structural stability of siRNA oligomers. Multimeric siRNA (Multi-siRNA) 115 and polymerized siRNA (Poly-siRNA) ${ }^{116}$, both of which were linked with disulfide bonds, were proposed by different groups. Multi-siRNA was prepared through the self-assembly of two complementary single-stranded siRNA dimers linked with flexible cross-linkers containing disulfide bonds at the $3^{\prime}$ ends. Gel electrophoresis showed a ladder-like migration pattern for multi-siRNA while naked siRNA had a single band, indicating that the assembled multi-siRNA consisted of stable siRNA oligomeric structures with a range of molecular weights in an aqueous solution. The multi-siRNA readily degrades in reductive environments and regenerates biologically active siRNA monomers. The multi-siRNA was formulated by LPEI $(25 \mathrm{kDa})$ to produce robust complexes having a relatively small size (a diameter of approximately $100 \mathrm{~nm}$ ) due to the increased charge density and flexible chains. In particular, the the AFM cantilever deflection of the multimeric siRNA/LPEI complexes in an aqueous solution was as strong as that of plasmid DNA/LPEI complexes, indicating the formation of compact complexes. GFP-expressing PC-3 tumors transfected by the multi-siRNA/LPEI complexes showed significantly inhibited in vivo GFP expression as compared to the level of inhibition by monomeric siRNA/LPEI complexes after an i.v. injection into mice. In a follow-up study, multi-siRNA structures were designed to incorporate two different siRNA sequences onto the same backbone for dual gene silencing. ${ }^{117}$ Two siRNA sequences containing thiol groups at both $3^{\prime}$ ends were randomly linked with reducible cross-linkers to form dual gene-silencing multi-siRNA (DGT multi-siRNA). DGT multi-siRNA containing apoptosis-related genes, $\mathrm{Bcl}-2$ and survivin siRNA, demonstrated a much higher level of apoptotic cell death for MCF cells compared to multi-siRNA targeting an individual gene with an equal amount of siRNA. Interestingly, dimeric siRNA conjugates in the form of only two siRNA molecules linked together via a cross-linker exhibited a gene silencing extent as efficient as that of multi-siRNA. ${ }^{118}$ The dimeric siRNA can also be constructed to enable combined gene therapy using a hetero-bifuncational cross-linker. The hetero-dimeric siRNA/LPEI (25 kDa) complexes showed the simultaneous inhibition of both GFP (41 $\%)$ and VEGF (53 \%) expression in cells. In addition, it was also shown that the length of the internal linkers in the dimeric siRNA greatly affected the stabilization of siRNA/LPEI complexes. ${ }^{119}$ The increased length of hydrophobic linkers increased the cellular uptake and gene silencing.

Recently, the same groups also demonstrated 
that AuNPs can be employed as a cross-linker to form multi-siRNA species for simultaneous molecular imaging and siRNA delivery. ${ }^{120}$ AuNP-mediated multi-siRNA was constructed by complementary hybridization between a single-stranded antisense and sense-siRNA immobilized AuNPs via Au-S chemisorption, which were readily reduced to produce free siRNA and AuNPs within the cell. By adjusting the feed molar ratio of siRNA to AuNP to about 1:3, the average number of siRNA per AuNP was modulated to about 2. The AuNP-mediated multi-siRNA/LPEI $(25 \mathrm{kDa})$ nanoparticles significantly improved the enzymatic stability of siRNA, cellular uptake, and gene-silencing efficiency compared to monomeric siRNA/LPEI nanoparticles. In particular, the computed tomography (CT) value of MDA-MB-435 cells transfected by the AuNP-mediated multi-siRNA/LPEI nanoparticles (129.6 HU) was more than 8-fold greater than that of monomeric siRNA/LPEI nanoparticles (15.8 HU). In a similar approach, poly-siRNA was synthesized from the polymerization of thiol-modified siRNA at both $5^{\prime}$ ends under mild oxidation conditions in HEPES buffer at $\mathrm{pH}$ 8.116 The resultant poly-siRNA was composed of different siRNA conjugates ranging from 50 to more than $300 \mathrm{bp}$ in length. The intensity of each gel band for poly-siRNA indicated that about $70 \%$ of poly-siRNA had more than $300 \mathrm{bp}$, indicating that poly-siRNA can have at least a 12-fold higher anionic charge compared to siRNA monomers. However, poly-siRNA was degraded and separated into siRNA monomers with RNAi activity after an addition of 8 $\mathrm{mM}$ DTT for $5 \mathrm{~h}$. The poly-siRNA/bPEI $(1.8 \mathrm{kDa})$ complexes reduced about $80 \%$ of red fluorescence protein (RFP) expression in B16F10-RFP cells. Recently, the same group investigated the efficiency of poly-siRNA with biocompatible cationic polymers for in vivo clinical applications. ${ }^{121}$ Poly-siRNA was formulated with thiol-grafted glycol chitosan (GC) through electrostatic interaction and chemical cross-linking, producing biocompatible nanoparticles encapsulating siRNA. It was conceivable that the electrostatic interaction of poly-siRNA with thiolated GC decreased the distance between thiol groups, allowing the thiolated GC to undergo intra- and inter-molecular cross-linking to form reducible and condensed nanoparticles. This reduced the amount of in vivo VEGF mRNA to $40 \%$ and suppressed tumor growth to $80 \%$ of the original value following $n$ i.v. injection into PC-3 tumor xenograft mice. Furthermore, mice injected with FPR-675-labeled poly-siRNA/GC complexes through the tail vein showed that the FPR signals in a tumor tissue were stronger than they were in any other organic tissues, including the liver, lung, spleen, kidney, and heart.
To obtain the simultaneous silencing of multiple genes, several recent studies have reported rationally designed RNA structures containing different target sequences. A variety of long single-stranded RNAs were hybridized to form a branched form of RNA having three- and four arms, called trimer RNA ${ }^{122}$ and tetramer RNA ${ }^{123}$, respectively, through complementary base paring. Each arm of the branched RNA contained different siRNA sequences, which can be readily cleaved by Dicer to produce free siRNA for RNAi-mediated gene silencing. Unlike naked siRNA with linear structures, the branched RNA possessed three- or four-way junctions to achieve a relatively high spatial charge density. Branched RNA incorporating different siRNAs for single/multiple target mRNA showed the simultaneous inhibition of target genes without the induction of an interferon response. These results indicated that multi-targeting RNA nanostructures can have a strong synergistic effect on enhancing the therapeutic potential to treat various diseases.

On the basis of the understanding that nucleases typically start by degrading at the loose ends of RNA, dumbbell-shaped nanocircular RNA was proposed for enhanced siRNA stability and prolonged RNAi activity. ${ }^{124,125}$ The RNA dumbbells consisted of a stem region encoding a siRNA sequence for target mRNA and two loops at both ends of the stem sequences. The loop positions were used to introduce various molecules such as nucleotides and polymers, which protected siRNA from enzymatic activity due to the terminal capping of the siRNA. In addition, termini-free RNA dumbbells were slowly digested by Dicer to form dsRNA species for the RNAi pathway as compared to unmodified linear dsRNA. This slow process provided RNA dumbbells with gene-silencing effects that were 1.5-fold more potent than those of unmodified siRNA after 5 days of transfection for NIH/3T3 cells.

The charge density and flexibility of siRNA have been shown to determine the structural stability of siRNA complexes upon interaction with cationic carriers. It is conceivable that the network structure of siRNA chains have favorable properties such as a high molecular weight, a high melting point, and good spatial charge density to form more stable, compact siRNA complexes with low-molecular-weight carriers. This attempt was initially reported as siRNA-based microhydrogel structures through the self-assembly of two complementary single-stranded Y-shaped siRNA molecules as building blocks (Figure 7). ${ }^{126}$ The network structure of microhydrogels showed a great number of nanoscale pores due to multiple Y-shaped junctions. In particular, the pore diameter and size of siRNA microhy- 
drogels were readily controlled by adjusting the concentrations of Y-shaped ss-siRNA. In addition, they were recognized and degraded by Dicer to generate biologically active siRNA species. Upon interaction with a low molecular cationic carrier (LPEI, $2.5 \mathrm{kDa}$ ), siRNA microhydrogels dramatically collapse to form stable siRNA complexes with a diameter of about 100 $\mathrm{nm}$. Interestingly, siRNA microhydrogel/LPEI complexes showed more highly enhanced gene silencing than multi-siRNA/LPEI complexes at a low siRNA dose. a

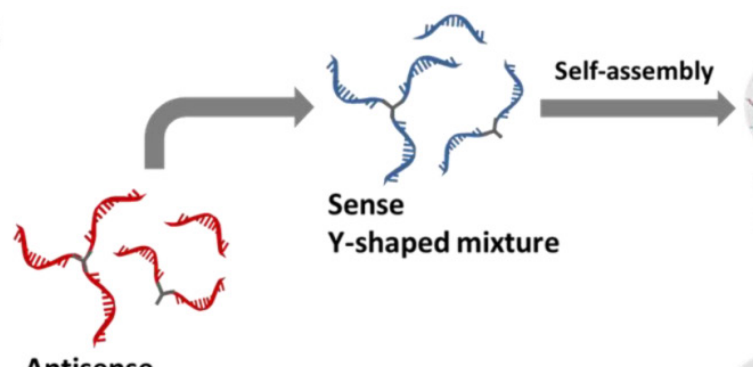

Antisense Y-shaped mixture
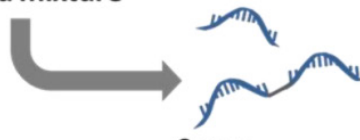

Self-assembly Sense dimeric mixture

b

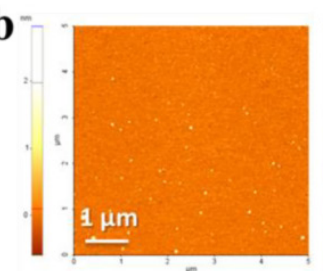

Monomeric siRNA

c

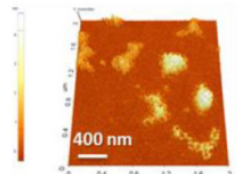

Monomeric siRNA/LPEI

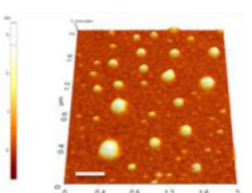

DY-siRNA/LPEI

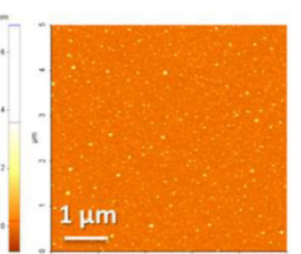

Multi-siRNA

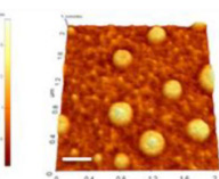

Multi-siRNA/LPEI

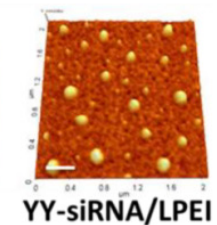

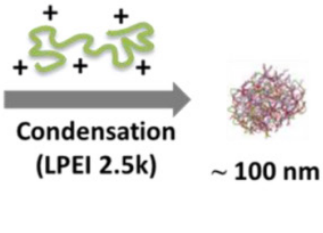

YY-siRNA

$(\sim 2 \mu \mathrm{m})$
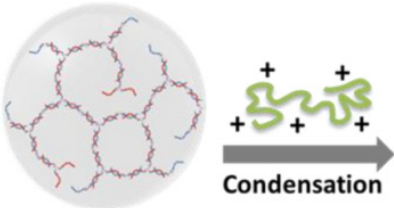

DY-siRNA

$(\sim 4 \mu \mathrm{m})$

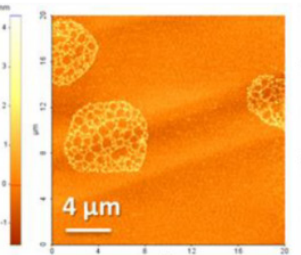

DY-siRNA

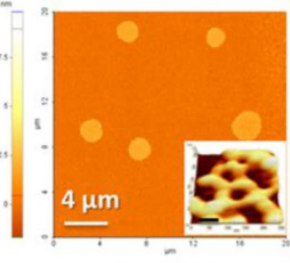

YY-siRNA

Figure 7. (a) Synthetic scheme of the preparation and polymeric condensation of siRNA microhydrogels: AFM images of (b) different siRNA-based structures and (c) their polymeric condensation with a cationic oligomer. The inset in YY-siRNA panel is a high-magnification 3D AFM images. (d) Dose-dependent GFP suppression effect of different siRNA/LPEI complexes in GFP-overexpressed MDA-MB-435 cells. Copyright 20II American Chemical Society.

Recently, oligonucleotide nanoparticles (ONPs) were prepared through the programmable self-assembly of DNA fragments and therapeutic siRNA for cell-specific siRNA delivery in vitro and in vivo. ${ }^{127}$ Six single-stranded DNA strands were assembled into a tetrahedron nanostructure containing 186 bp and 20-nucleotide overhangs in the middle of each edge. The overhang strands were complementary to the overhang of the siRNA molecules, enabling six siRNAs to attach to one tetrahedral ONP. Dynamic light scattering found a hydrodynamic diameter of about $28 \mathrm{~nm}$ with a narrow size distribution. The ONPs showed a much longer half-life time (about 24 min) in blood circulation than naked siRNA (about 6 $\mathrm{min}$ ). To achieve tumor-specific targeting effects, folic acids (FA) were introduced to ONPs. The FA-conjugated ONPs induced GFP suppression in excess of $60 \%$ in human nasopharyngeal cells (KB) cells expressing a high number of folate receptors. Interestingly, gene silencing efficiency depended upon the spatial orientation and the density of the targeted ligands on the ONP surface. FA-ONPs with 
anti-luciferase siRNA induced up to a $60 \%$ decrease in the bioluminescent intensity at the tumor site of luciferase-expressing KB xenografts via both tail-vein and intratumoral injections.

More recently, a unique class of a highly condensed siRNA structure with RNAi activity was suggested via an enzymatic RNA polymerization process known as rolling circle transcription (RCT). ${ }^{128}$ Using the RCT reaction of a DNA template, extremely long polymers of RNA hairpins containing siRNA sequences were synthesized. The RNA polymers served as building blocks and were spontaneously assembled to generate a crystal-like ordered RNA sheet. In addition, some of the RNA sheets continuously collapsed to form densely packed sponge-like spherical microparticles with a diameter of about 2 $\mu \mathrm{m}$. The conformation of RNAi-microsponges remained stable at temperatures as high as $150{ }^{\circ} \mathrm{C}$ due to their crystalline structures. The RNAi-microsponges were composed of siRNA precursors, which were degraded by Dicer processing to regenerate free siRNA molecules for RNAi effects. The LPEI-coated (25 kDa) microsponges efficiently inhibited the firefly luciferase expression in T22 cells in vitro and mouse tumors in vivo after an intratumoral injection, whereas no significant change was observed for a control RNAi-microsponge/LPEI complex.

\section{Conclusions and Outlook}

The discovery of RNAi has been considered one of the most exciting and significant medical breakthroughs, as RNAi activity selectively silences any genes in the genome. In particular, siRNA-mediated gene silencing has great potential in the treatment of cancers and gene-related diseases in mammalian cells. However, a safe and efficient delivery system of siRNA into the cytoplasm of targeted cells remains as a bottleneck for clinical applications. A key challenge in siRNA therapy is the issue of delivery. The rapid degradation and renal clearance of siRNA in the blood stream make it difficult to remain stable until arrival at a target site. Due to the low pharmaceutical property of siRNA, the current RNAi therapeutics in clinical trials has been focused on the direct administration of siRNA into the target tissues such as eyes, lung, and brain. This local delivery has demonstrated the successful gene silencing in animal models. However, it is highly demanded to develop new siRNA delivery systems for in vivo targeting of specific cells and tissues. To address this challenge, a wide variety of siRNA delivery systems have been suggested, and some of them have showed promising preclinical results. End-modified siRNA provided efficient conjugation with various biomolecules, such as functional polymers, targeted ligands, and imaging probes. The conjugation sites and types of siRNA play important roles in gene silencing and immune responses. Liposomal encapsulation technology has improved the half-life of siRNA in the bloodstream. In addition, multifunctional and biocompatible liposomes encapsulating siRNA were extensively studied for clinical applications over the last few years. Significant advances in the surface modification, functionalization, and conjugation of metallic core nanoparticles have been made with multifunctional nanoparticles for siRNA delivery and imaging in vivo and in vitro. Recently, siRNA-based nanostructures have provided a new opportunity for the fabrication of stable complexes with low-molecular-weight cationic carriers due to the increased spatial charge density and structural flexibility. This approach shows a synergetic effect, demonstrating a high loading efficiency of siRNA per nanoparticle, low cytotoxicity, and prolonged RNAi activity. Although a variety of siRNA carriers have been suggested, the stability and efficiency of siRNA delivery systems must be improved for practical applications.

Table 3. The current progress on RNAi therapeutics in clinical trials.

\begin{tabular}{|c|c|c|c|c|}
\hline Drug & Sponsor & Target gene & Diseases & Status \\
\hline PF-655 & Quark Pharm., Inc. & RTP801 & Age-related macular degeneration & Phase II \\
\hline QPI-1007 & Quark Pharm., Inc. & Caspase 2 & Non-arteritic anterior ischemic optic neuropathy & Phase II \\
\hline QPI-1002/I5NP & Quark Pharm., Inc. & p53 & Acute kidney injury & Phase I / II \\
\hline ALN-RSV01 & Alnylam Pharm. & RSV nucleocapsid & Bronchiolitis obliterans syndrome & $\begin{array}{l}\text { Completed, } \\
\text { Phase II }\end{array}$ \\
\hline AGN211745 & Sirna Therapeutics, Inc. & VEGFR1 & Age-related macular degeneration & Terminated, Phase II \\
\hline SYL040012 & Sylentis & $\beta 2$ adrenergic receptor & Ocular hypertension & Completed, Phase II \\
\hline Bevasiranib & Opko Health, Inc. & VEGF & Age-related macular degeneration & Terminated, Phase III \\
\hline pHIV7-shI-TAR-CCR5RZ & City of Hope Medical Center & $\begin{array}{l}\text { HIV Tat protein, HIV TAR } \\
\text { RNA, human CCR5 }\end{array}$ & Human immunodeficiency virus (HIV) & Terminated, Phase 0 \\
\hline Atu027-I-02 & Silence Therapeutics & Protein kinase N3 & Ancreatic cancer & Phase I/II \\
\hline NCT00672542 & Scott Pruitt & LMP2, LMP7, and MECL1 & Metastatic melanoma & Completed, Phase I \\
\hline SPC2996 & Santaris Pharm. & $\mathrm{Bcl}-2$ & Chronic lymphocytic leukemia & Completed, Phase I, II \\
\hline
\end{tabular}


Table 4. Engineered siRNA-based structures with RNAi activity. DTME (Dithiobismaleimidoethane), BMPEG 2 (I,8-Bismaleimido-diethyleneglycol), and TMEA (Tris(2-maleimidoethyl)amine). Copyright 2012 Nature Publishing Group.

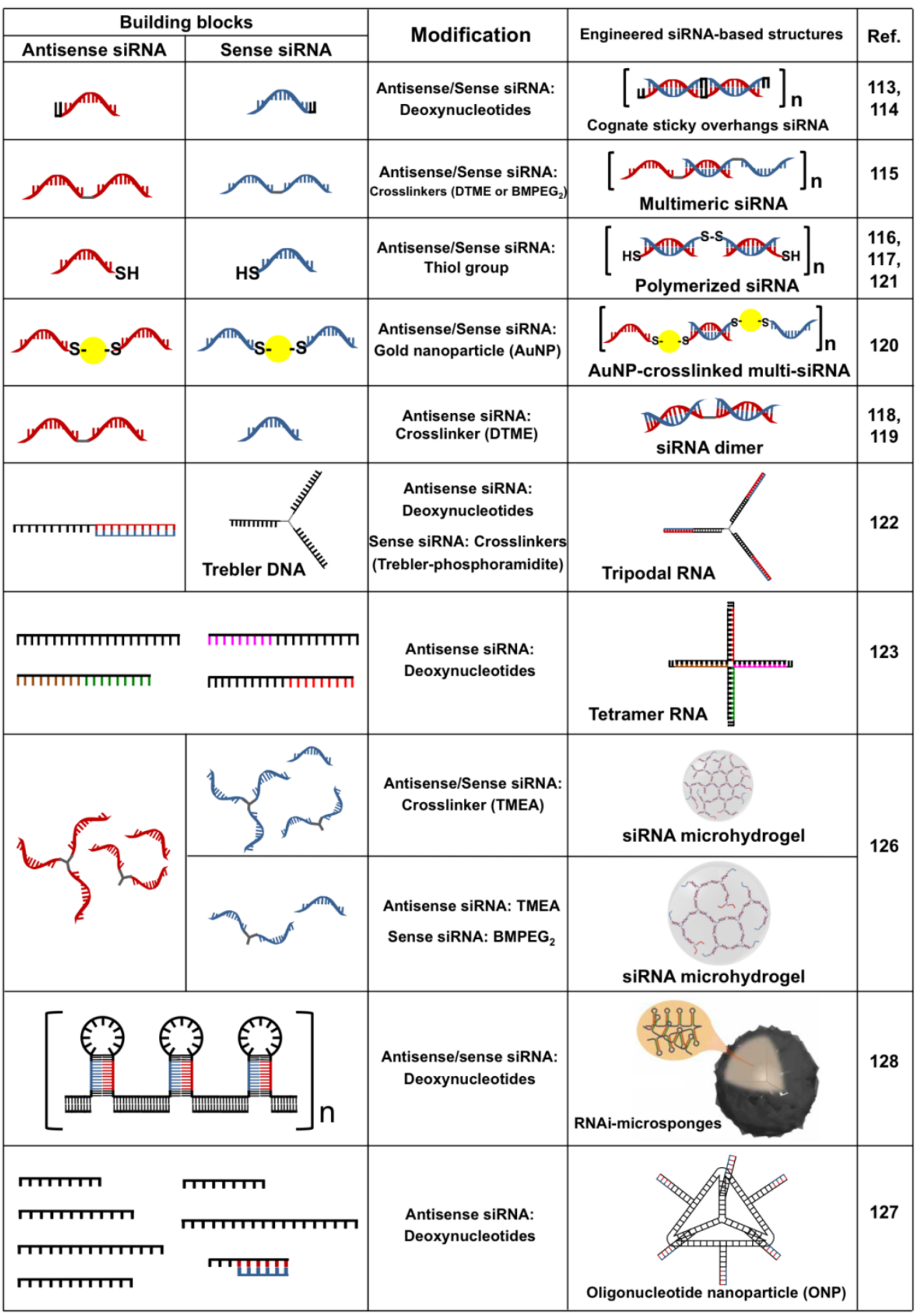

\section{Acknowledgements}

This research was financially supported from the Ministry of Health, Welfare and Family Affairs of Republic of Korea (A111552).

\section{Competing Interests}

The authors have declared that no competing interest exists.

\section{References}

1. Fire A, Xu S, Montgomery MK, Kostas SA, Driver SE, Mello CC. Potent and specific genetic interference by double-stranded RNA in Caenorhabditis elegans. Nature 1998;391:806-11.

2. Lakshman DK, Jian J, Tavantzis SM. A double-stranded RNA element from a hypovirulent strain of Rhizoctonia solani occurs in DNA form and is genetically related to the pentafunctional AROM protein of the shikimate pathway. Proc Natl Acad Sci U S A 1998;95:6425-9.

3. Clemens JC, Worby CA, Simonson-Leff N, Muda M, Maehama T, Hemmings BA, et al. Use of double-stranded RNA interference in Drosophila cell lines to dissect signal transduction pathways. Proc Natl Acad Sci U S A 2000;97:6499-503. 
4. Waterhouse PM, Graham MW, Wang MB. Virus resistance and gene silencing in plants can be induced by simultaneous expression of sense and antisense RNA. Proc Natl Acad Sci U S A 1998;95:13959-64.

5. Alexopoulou L, Holt AC, Medzhitov R, Flavell RA. Recognition of double-stranded RNA and activation of NF-kappaB by Toll-like receptor 3 . Nature 2001;413:732-8.

6. Caplen NJ, Parrish S, Imani F, Fire A, Morgan RA. Specific inhibition of gene expression by small double-stranded RNAs in invertebrate and vertebrate systems. Proc Natl Acad Sci U S A 2001;98:9742-7.

7. Macrae IJ, Zhou K, Li F, Repic A, Brooks AN, Cande WZ, et al. Structural basis for double-stranded RNA processing by Dicer. Science 2006;311:195-8.

8. Vermeulen A, Behlen L, Reynolds A, Wolfson A, Marshall WS, Karpilow J, et al. The contributions of dsRNA structure to Dicer specificity and efficiency. RNA 2005;11:674-82.

9. Agrawal N, Dasaradhi PV, Mohmmed A, Malhotra P, Bhatnagar RK, Mukherjee SK. RNA interference: biology, mechanism, and applications. Microbiol Mol Biol Rev 2003;67:657-85.

10. Elbashir SM, Harborth J, Lendeckel W, Yalcin A, Weber K, Tuschl T. Duplexes of 21-nucleotide RNAs mediate RNA interference in cultured mammalian cells. Nature 2001;411:494-8.

11. Schwarz DS, Hutvagner G, Haley B, Zamore PD. Evidence that siRNAs function as guides, not primers, in the Drosophila and human RNAi pathways. Mol Cell 2002;10:537-48.

12. Tan SJ, Kiatwuthinon P, Roh YH, Kahn JS, Luo D. Engineering Nanocarriers for siRNA Delivery. Small 2011;7:841-56.

13. Oh YK and Park TG. siRNA delivery systems for cancer treatment. Adv Drug Deliv Rev 2009;61:850-62

14. Turner JJ, Jones SW, Moschos SA, Lindsay MA, Gait MJ. MALDI-TOF mass spectral analysis of siRNA degradation in serum confirms an RNAse A-like activity. Mol Biosyst 2007;3:43-50.

15. Chen $\mathrm{M}, \mathrm{Du} \mathrm{O}$, Zhang HY, Wahlestedt $\mathrm{C}$, Liang Z. Vector-based siRNA delivery strategies for high-throughput screening of novel target genes. J RNAi Gene Silencing 2005;1:5-11.

16. Hosono T, Mizuguchi H, Katayama K, Xu ZL, Sakurai F, Ishii-Watabe A, et al. Adenovirus vector-mediated doxycycline-inducible RNA interference. Hum Gene Ther 2004;15:813-9.

17. Lee NS, Dohjima T, Bauer G, Li H, Li MJ, Ehsani A, et al. Expression of small interfering RNAs targeted against HIV-1 rev transcripts in human cells. Nature Biotech 2002;20:500-5.

18. Gao Y, Liu XL, Li XR. Research progress on siRNA delivery with nonviral carriers. Int J Nanomedicine 2011:6:1017-25.

19. Elsabahy M, Nazarali A, Foldvari M. Non-viral nucleic acid delivery: key challenges and future directions. Curr Drug Deliv 2011;8:235-44

20. Lee K, Oh MH, Lee MS, Nam YS, Park TG, Jeong JH. Stabilized calcium phosphate nano-aggregates using a dopa-chitosan conjugate for gene delivery. Int J Pharm 2013; 445:196-202.

21. Li SD, Huang L. Targeted delivery of siRNA by nonviral vectors: lessons learned from recent advances. Curr Opin Investig Drugs 2008;9:1317-23.

22. Thomas M, Klibanov AM. Non-viral gene therapy: polycation-mediated DNA delivery. Appl Microbiol Biotechnol 2003;62:27-34.

23. Kwok A, Hart SL. Comparative structural and functional studies of nanoparticle formulations for DNA and siRNA delivery. Nanomedicine 2011;7:210-9.

24. Park TG, Jeong JH, Kim SW. Current status of polymeric gene delivery systems. Adv Drug Deliv Rev 2006;58:467-86.

25. Gary DJ, Puri N, Won YY. Polymer-based siRNA delivery: perspectives on the fundamental and phenomenological distinctions from polymer-based DNA delivery. J Control Release 2007;121:64-73.

26. Peng Q, Zhong Z, Zhuo R. Disulfide cross-linked polyethylenimines (PEI) prepared via thiolation of low molecular weight PEI as highly efficient gene vectors. Bioconjug Chem 2008;19:499-506.

27. Son S, Namgung R, Kim J, Singha K, Kim WJ. Bioreducible polymers for gene silencing and delivery. Acc Chem Res 2012;45:1100-12.

28. Singh N, Agrawal A, Leung AK, Sharp PA, Bhatia SN. Effect of nanoparticle conjugation on gene silencing by RNA interference. J Am Chem Soc 2010;132:8241-3.

29. Watts JK, Deleavey GF, Damha MJ. Chemically modified siRNA: tools and applications. Drug Discov Today 2008;13:842-55.

30. Al-Abd AM, Lee SH, Kim SH, Cha JH, Park TG, Lee SI, et al. Penetration and efficacy of VEGF siRNA using polyelectrolyte complex micelles in a human solid tumor model in-vitro. J Control Release 2009;137:130-5.

31. Kim SH, Jeong JH, Lee SH, Kim SW, Park TG. PEG conjugated VEGF siRNA for anti-angiogenic gene therapy. J Control Release 2006;116:123-9.

32. Kim SH, Jeong JH, Lee SH, Kim SW, Park TG. Local and systemic delivery of VEGF siRNA using polyelectrolyte complex micelles for effective treatment of cancer. J Control Release 2008;129:107-16.

33. Choi SW, Lee SH, Mok H, Park TG. Multifunctional siRNA delivery system: polyelectrolyte complex micelles of six-arm PEG conjugate of siRNA and cell penetrating peptide with crosslinked fusogenic peptide. Biotechnol Prog 2010;26:57-63.

34. Jung S, Lee SH, Mok H, Chung HJ, Park TG. Gene silencing efficiency of siRNA-PEG conjugates: effect of PEGylation site and PEG molecular weight. J Control Release 2010;144:306-13.

35. Oishi M, Nagasaki Y, Itaka K, Nishiyama N, Kataoka K. Lactosylated poly(ethylene glycol)-siRNA conjugate through acid-labile beta-thiopropionate linkage to construct $\mathrm{pH}$-sensitive polyion complex micelles achieving enhanced gene silencing in hepatoma cells. J Am Chem Soc 2005;127:1624-5.

36. Dohmen C, Frohlich T, Lachelt U, Rohl I, Vornlocher HP, Hadwiger P, et al. Defined Folate-PEG-siRNA Conjugates for Receptor-specific Gene Silencing. Mol Ther Nucleic Acids 2012;1:e7.

37. Nothisen M, Kotera M, Voirin E, Remy JS, Behr JP. Cationic siRNAs provide carrier-free gene silencing in animal cells. J Am Chem Soc 2009;131:17730-1.

38. Perche P, Nothisen M, Bagilet J, Behr JP, Kotera M, Remy JS. Cell-penetrating cationic siRNA and lipophilic derivatives efficient at nanomolar concentrations in the presence of serum and albumin. J Control Release 2013;170:92-8

39. Rozema DB, Lewis DL, Wakefield DH, Wong SC, Klein JJ, Roesch PL, et al. Dynamic PolyConjugates for targeted in vivo delivery of siRNA to hepatocytes. Proc Natl Acad Sci U S A 2007;104:12982-7.

40. Meyer M, Dohmen C, Philipp A, Kiener D, Maiwald G, Scheu C, et al. Synthesis and biological evaluation of a bioresponsive and endosomolytic siRNA-polymer conjugate. Mol Pharm 2009;6:752-62.

41. Jeong JH, Park TG. Novel polymer-DNA hybrid polymeric micelles composed of hydrophobic poly(D,L-lactic-co-glycolic acid) and hydrophilic oligonucleotides. Bioconjug Chem 2001;12:917-23.

42. Nam YS and Park TG. Protein loaded biodegradable microspheres based on PLGA-protein bioconjugates. J Microencapsul 1999;16:625-37.

43. Lee JY, Lee SH, Oh MH, Kim JS, Park TG, Nam YS. Prolonged gene silencing by siRNA/chitosan-g-deoxycholic acid polyplexes loaded within biodegradable polymer nanoparticles. J Control Release 2012; 162:407-13.

44. Oh JE, Nam YS, Lee KH, Park TG. Conjugation of drug to poly(D,L-lactic-co-glycolic acid) for controlled release from biodegradable microspheres. J Control Release 1999;57:269-80.

45. Lee SH, Mok H, Lee Y, Park TG. Self-assembled siRNA-PLGA conjugate micelles for gene silencing. J Control Release 2011;152:152-8.

46. Park K, Yang JA, Lee MY, Lee H, Hahn SK. Reducible Hyaluronic Acid-siRNA Conjugate for Target Specific Gene Silencing. Bioconjug Chem 2013; 24:1201-120.

47. Kim JS, Oh MH, Park JY, Park TG, Nam YS. Protein-resistant, reductively dissociable polyplexes for in vivo systemic delivery and tumor-targeting of siRNA. Biomaterials 2013; 34:2370-9.

48. Namgung R and Kim WJ. A highly entangled polymeric nanoconstruct assembled by siRNA and its reduction-triggered siRNA release for gene silencing. Small 2012;8:3209-019.

49. Hong CA, Kim JS, Lee SH, Kong WH, Park TG, Mok H, et al. Reductively Dissociable siRNA-Polymer Hybrid Nanogels for Efficient Targeted Gene Silencing. Adv Funct Mater 2013;23:316-22.

50. Maurer N, Fenske DB, Cullis PR. Developments in liposomal drug delivery systems. Expert Opin Biol Ther 2001;1:923-47.

51. Buyens K, De Smedt SC, Braeckmans K, Demeester J, Peeters L, van Grunsven LA, et al. Liposome based systems for systemic siRNA delivery: stability in blood sets the requirements for optimal carrier design. J Control Release 2012;158:362-70.

52. Oh MH, Kim JS, Lee JY, Park TG, Nam YS. Radio-opaque theranostic nanoemulsions with synergistic anti-cancer activity of paclitaxel and Bcl-2 iRNA. RSC Adv 2013;3: 14642-51.

53. Zhang C, Tang N, Liu X, Liang W, Xu W, Torchilin VP. siRNA-containing liposomes modified with polyarginine effectively silence the targeted gene. J Control Release 2006;112:229-39.

54. Wu SY, Putral LN, Liang M, Chang HI, Davies NM, McMillan NA. Development of a novel method for formulating stable siRNA-loaded lipid particles for in vivo use. Pharm Res 2009:26:512-22.

55. Landen CN, Jr., Chavez-Reyes A, Bucana C, Schmandt R, Deavers MT, Lopez-Berestein G, et al. Therapeutic EphA2 gene targeting in vivo using neutral liposomal small interfering RNA delivery. Cancer Res 2005;65:6910-8.

56. Mokhtarieh AA, Cheong S, Kim S, Chung BH, Lee MK. Asymmetric liposome particles with highly efficient encapsulation of siRNA and without nonspecific cell penetration suitable for target-specific delivery. Biochim Biophys Acta 2012;1818:1633-41.

57. Chono S, Li SD, Conwell CC, Huang L. An efficient and low immunostimulatory nanoparticle formulation for systemic siRNA delivery to the tumor. J Control Release 2008;131:64-9.

58. Wang Y, Zhang L, Guo S, Hatefi A, Huang L. Incorporation of histone derived recombinant protein for enhanced disassembly of core-membrane structured liposomal nanoparticles for efficient siRNA delivery. J Control Release 2013:172:179-89.

59. Peer D, Park EJ, Morishita Y, Carman CV, Shimaoka M. Systemic leukocyte-directed siRNA delivery revealing cyclin D1 as an anti-inflammatory target. Science 2008;319:627-30.

60. Morrissey DV, Lockridge JA, Shaw L, Blanchard K, Jensen K, Breen W, et al Potent and persistent in vivo anti-HBV activity of chemically modified siRNAs. Nature Biotech 2005;23:1002-7.

61. Zimmermann TS, Lee AC, Akinc A, Bramlage B, Bumcrot D, Fedoruk MN, et al. RNAi-mediated gene silencing in non-human primates. Nature 2006;441:111-4

62. Akinc A, Goldberg M, Qin J, Dorkin JR, Gamba-Vitalo C, Maier M, et al Development of lipidoid-siRNA formulations for systemic delivery to the liver. Mol Ther 2009;17:872-9. 
63. Akinc A, Zumbuehl A, Goldberg M, Leshchiner ES, Busini V, Hossain N, et al. A combinatorial library of lipid-like materials for delivery of RNAi therapeutics. Nature Biotech 2008;26:561-9.

64. Love KT, Mahon KP, Levins CG, Whitehead KA, Querbes W, Dorkin JR, et al Lipid-like materials for low-dose, in vivo gene silencing. Proc Natl Acad Sci U S A 2010;107:1864-9.

65. Sperling RA, Rivera Gil P, Zhang F, Zanella M, Parak WJ. Biological applications of gold nanoparticles. Chem Soc Rev 2008;37:1896-908.

66. Oishi M, Nakaogami J, Ishii T, Nagasaki Y. Smart PEGylated Gold Nanoparticles for the Cytoplasmic Delivery of siRNA to Induce Enhanced Gene Silencing. Chem Lett 2006;35:1046-7.

67. Lee JS, Green JJ, Love KT, Sunshine J, Langer R, Anderson DG. Gold, poly(beta-amino ester) nanoparticles for small interfering RNA delivery. Nano Lett 2009;9:2402-6.

68. Giljohann DA, Seferos DS, Prigodich AE, Patel PC, Mirkin CA. Gene regulation with polyvalent siRNA-nanoparticle conjugates. J Am Chem Soc 2009;131:2072-3.

69. Lee SH, Bae KH, Kim SH, Lee KR, Park TG. Amine-functionalized gold nanoparticles as non-cytotoxic and efficient intracellular siRNA delivery carriers. Int J Pharm 2008;364:94-101.

70. Kim ST, Chompoosor A, Yeh YC, Agasti SS, Solfiell DJ, Rotello VM. Dendronized gold nanoparticles for siRNA delivery. Small 2012;8:3253-6.

71. Song WJ, Du JZ, Sun TM, Zhang PZ, Wang J. Gold nanoparticles capped with polyethyleneimine for enhanced siRNA delivery. Small 2010;6:239-46.

72. Lee Y, Lee SH, Kim JS, Maruyama A, Chen X, Park TG. Controlled synthesis of PEI-coated gold nanoparticles using reductive catechol chemistry for siRNA delivery. J Control Release 2011;155:3-10.

73. A, Zaky A, Liebl R, Rachel R, Goepferich A, Breunig M. Layer-by-layer assembled gold nanoparticles for siRNA delivery. Nano Lett 2009;9:2059-64.

74. Guo S, Huang Y, Jiang Q, Sun Y, Deng L, Liang Z, et al. Enhanced gene delivery and siRNA silencing by gold nanoparticles coated with charge-reversal polyelectrolyte. ACS Nano 2010;4:5505-11.

75. Han L, Zhao J, Zhang X, Cao W, Hu X, Zou G, et al. Enhanced siRNA Delivery and Silencing Gold-Chitosan Nanosystem with Surface Charge-Reversal Polymer Assembly and Good Biocompatibility. ACS Nano 2012;6:7340-51.

76. Lee SK, Han MS, Asokan S, Tung CH. Effective gene silencing by multilayered siRNA-coated gold nanoparticles. Small 2011;7:364-70.

77. Lee MY, Park SJ, Park K, Kim KS, Lee H, Hahn SK. Target-specific gene silencing of layer-by-layer assembled gold-cysteamine/siRNA/PEI/HA nanocomplex. ACS Nano 2011;5:6138-47.

78. Braun GB, Pallaoro A, Wu G, Missirlis D, Zasadzinski JA, Tirrell M, et al. Laser-Activated Gene Silencing via Gold Nanoshell-siRNA Conjugates. ACS Nano 2009;3:2007-15.

79. Lu W, Zhang G, Zang R, Flores ll LG, Huang Q, Gelovani JG, Li C. Tumor Site-Specific Silencing of NF-kB p65 by Targeted Hollow Gold Nanosphere-Mediated Photothermal Transfection. Cancer Res 2010;70:3177-88.

80. Huschka R, Barhoumi A, Liu Q, Roth JA, Ji L, Halas NJ. Gene silencing by gold nanoshell-mediated delivery and laser-triggered release of antisense oligonucleotide and siRNA. ACS Nano 2012;6:7681-91.

81. Sosnovik DE, Nahrendorf M, Weissleder R. Magnetic nanoparticles for MR imaging: agents, techniques and cardiovascular applications. Basic Res Cardiol 2008;103:122-30.

82. Liu G, Swierczewska M, Lee S, Chen X. Functional Nanoparticles for Molecular Imaging Guided Gene Delivery. Nano Today 2010;5:524-39.

83. McBain SC, Yiu HH, Dobson J. Magnetic nanoparticles for gene and drug delivery. Int J Nanomedicine 2008;3:169-80.

84. Boyer C, Priyanto P. Davis TP. Pissuwan D. Bulmus V. Kavallaris M. Teoh WY. Amal R. Carroll M. Woodward R. Pierre TS. Anti-fouling magnetic nanoparticles for siRNA delivery. J Mater Chem 2009;20:255-65.

85. Medarova Z, Pham W, Farrar C, Petkova V, Moore A. In vivo imaging of siRNA delivery and silencing in tumors. Nature Med 2007:13:372-7.

86. Maeda H, Wu J, Sawa T, Matsumura Y, Hori K. Tumor vascular permeability and the EPR effect in macromolecular therapeutics: a review. J Control Release 2000;65:271-84.

87. Agrawal A, Min DH, Singh N, Zhu H, Birjiniuk A, von Maltzahn G, et al. Functional delivery of siRNA in mice using dendriworms. ACS Nano 2009;3:2495-504.

88. Namiki Y, Namiki T, Yoshida H, Ishii Y, Tsubota A, Koido S, et al. A novel magnetic crystal-lipid nanostructure for magnetically guided in vivo gene delivery. Nature Nanotech 2009;4:598-606.

89. Lee JH, Lee $\mathrm{K}$, Moon SH, Lee $\mathrm{Y}$, Park TG, Cheon J. All-in-one target-cell-specific magnetic nanoparticles for simultaneous molecular imaging and siRNA delivery. Angew Chem Int Ed Engl 2009;48:4174-9.

90. Veiseh O, Kievit FM, Fang C, Mu N, Jana S, Leung MC, et al. Chlorotoxin bound magnetic nanovector tailored for cancer cell targeting, imaging, and siRNA delivery. Biomaterials 2010;31:8032-42.

91. Veiseh O, Kievit FM, Mok H, Ayesh J, Clark C, Fang C, et al. Cell transcytosing poly-arginine coated magnetic nanovector for safe and effective siRNA delivery. Biomaterials 2011;32:5717-25.

92. Mok H, Veiseh O, Fang C, Kievit FM, Wang FY, Park JO, et al. pH-Sensitive siRNA nanovector for targeted gene silencing and cytotoxic effect in cancer cells. Mol Pharm 2010;7:1930-9.
93. Namiki Y, Namiki T, Yoshida H, Ishii Y, Tsubota A, Koido S, et al. A novel magnetic crystal-lipid nanostructure for magnetically guided in vivo gene delivery. Nature Nanotech 2009;4:598-606.

94. Lee K, Bae KH, Lee Y, Lee SH, Ahn CH, Park TG. Pluronic/polyethylenimine shell crosslinked nanocapsules with embedded magnetite nanocrystals for magnetically triggered delivery of siRNA. Macromol Biosci 2010;10:239-45.

95. Park JW, Bae KH, Kim C, Park TG. Clustered magnetite nanocrystals cross-linked with PEI for efficient siRNA delivery. Biomacromolecules 2011;12:457-65

96. Liu G, Xie J, Zhang F, Wang Z, Luo K, Zhu L, et al. N-Alkyl-PEI-functionalized iron oxide nanoclusters for efficient siRNA delivery. Small 2011:7:2742-9.

97. Juzenas P, Chen W, Sun YP, Coelho MA, Generalov R, Generalova N, et al. Quantum dots and nanoparticles for photodynamic and radiation therapies of cancer. Adv Drug Deliv Rev 2008;60:1600-14.

98. Sanvicens N, Marco MP. Multifunctional nanoparticles--properties and prospects for their use in human medicine. Trends Biotechnol 2008;26:425-33.

99. Derfus AM, Chen AA, Min DH, Ruoslahti E, Bhatia SN. Targeted quantum dot conjugates for siRNA delivery. Bioconjug Chem 2007;18:1391-6.

100. Jung J, Solanki A, Memoli KA, Kamei K, Kim H, Drahl MA, et al. Selective inhibition of human brain tumor cells through multifunctional quantum-dot-based siRNA delivery. Angew Chem Int Ed Engl 2010;49:103-7.

101. Yezhelyev MV, Qi L, O'Regan RM, Nie S, Gao X. Proton-sponge coated quantum dots for siRNA delivery and intracellular imaging. J Am Chem Soc 2008;130:9006-12.

102. Tan WB, Jiang S, Zhang Y. Quantum-dot based nanoparticles for targeted silencing of HER2/neu gene via RNA interference. Biomaterials 2007;28:1565-71.

103. Lee H, Kim IK, Park TG. Intracellular trafficking and unpacking of siRNA/quantum dot-PEI complexes modified with and without cell penetrating peptide: confocal and flow cytometric FRET analysis. Bioconjug Chem 2010;21:289-95.

104. Bagalkot V, Gao X. siRNA-aptamer chimeras on nanoparticles: preserving targeting functionality for effective gene silencing. ACS Nano 2011;5:8131-9.

105. Qi L and Gao X. Quantum dot-amphipol nanocomplex for intracellular delivery and real-time imaging of siRNA. ACS Nano 2008;2:1403-10.

106. Ortiz Mellet C, Garcia Fernandez JM, Benito JM. Cyclodextrin-based gene delivery systems. Chem Soc Rev 2011;40:1586-608.

107. Tiwari G, Tiwari R, Rai AK. Cyclodextrins in delivery systems: Applications. J Pharm Bioallied Sci 2010;2:72-9.

108. Li JM, Zhao MX, Su H, Wang YY, Tan CP, Ji LN, et al. Multifunctional quantum-dot-based siRNA delivery for HPV18 E6 gene silence and intracellular imaging. Biomaterials 2011;32:7978-87.

109. Li JM, Wang YY, Zhao MX, Tan CP, Li YQ, Le XY, et al. Multifunctional QD-based co-delivery of siRNA and doxorubicin to HeLa cells for reversal of multidrug resistance and real-time tracking. Biomaterials 2012;33:2780-90.

110. Ashley CE, Carnes EC, Phillips GK, Durfee PN, Buley MD, Lino CA, et al. Cell-specific delivery of diverse cargos by bacteriophage MS2 virus-like particles. ACS Nano 2011;5:5729-45.

111. Bae KH, Lee JY, Lee SH, Park TG, Nam YS. Optically traceable solid lipid nanoparticles loaded with siRNA and paclitaxel for synergistic chemotherapy with in situ imaging. Adv Healthc Mater. 2013;2:576-84.

112. Lee SH, Chung B, Park TG, Mok H, Nam YS. Small interfering RNA (siRNA)-based functional micro/nanostructures for efficient and selective gene silencing. Acc Chem Res 2012;45:1014-25.

113. Bolcato-Bellemin AL, Bonnet ME, Creusat G, Erbacher P, Behr JP. Sticky overhangs enhance siRNA-mediated gene silencing. Proc Natl Acad Sci U S A 2007;104:16050-5.

114. Liu X, Liu C, Laurini E, Posocco P, Pricl S, Qu F, et al. Efficient delivery of sticky siRNA and potent gene silencing in a prostate cancer model using a generation 5 triethanolamine-core PAMAM dendrimer. Mol Pharm 2012;9:470-81.

115. Mok H, Lee SH, Park JW, Park TG. Multimeric small interfering ribonucleic acid for highly efficient sequence-specific gene silencing. Nature Mater 2010;9:272-8.

116. Lee SY, Huh MS, Lee S, Lee SJ, Chung H, Park JH, et al. Stability and cellular uptake of polymerized siRNA (poly-siRNA)/polyethylenimine (PEI) complexes for efficient gene silencing. J Control Release 2010;141:339-46.

117. Lee SH, Mok H, Jo S, Hong CA, Park TG. Dual gene targeted multimeric siRNA for combinatorial gene silencing. Biomaterials 2011;32:2359-68.

118. Chung HJ, Hong CA, Lee SH, Jo SD, Park TG. Reducible siRNA dimeric conjugates for efficient cellular uptake and gene silencing. Bioconjug Chem 2011;22:299-306.

119. Jo SD, Kim JS, Joe CO, Mok H, Nam YS. Small interfering RNA nunchucks with a hydrophobic linker for efficient intracellular delivery. Macromol Biosci. 2014;14:195-201.

120. Kong WH, Bae KH, Hong CA, Lee $Y$, Hahn SK, Park TG. Multimerized siRNA cross-linked by gold nanoparticles. Bioconjug Chem 2011;22:1962-9.

121. Lee SJ, Huh MS, Lee SY, Min S, Lee S, Koo H, et al. Tumor-homing poly-siRNA/glycol chitosan self-cross-linked nanoparticles for systemic siRNA delivery in cancer treatment. Angew Chem Int Ed Engl 2012;51:7203-7.

122. Chang CI, Lee TY, Kim S, Sun X, Hong SW, Yoo JW, et al. Enhanced intracellular delivery and multi-target gene silencing triggered by tripodal RNA structures. J Gene Med 2012;14:138-46.

123. Nakashima Y, Abe H, Abe N, Aikawa K, Ito Y. Branched RNA nanostructures for RNA interference. Chem Commun 2011;47:8367-9. 
124. Abe N, Abe H, Ito Y. Dumbbell-shaped nanocircular RNAs for RNA interference. J Am Chem Soc 2007;129:15108-9.

125. Abe N, Abe H, Nagai C, Harada M, Hatakeyama H, Harashima H, et al. Synthesis, structure, and biological activity of dumbbell-shaped nanocircular RNAs for RNA interference. Bioconjug Chem 2011;22:2082-92.

126. Hong CA, Lee SH, Kim JS, Park JW, Bae KH, Mok H, et al. Gene silencing by siRNA microhydrogels via polymeric nanoscale condensation. J Am Chem Soc 2011;133:13914-7.

127. Lee H, Lytton-Jean AK, Chen Y, Love KT, Park AI, Karagiannis ED, et al. Molecularly self-assembled nucleic acid nanoparticles for targeted in vivo siRNA delivery. Nature Nanotech 2012;7:389-93.

128. Lee JB, Hong J, Bonner DK, Poon Z, Hammond PT. Self-assembled RNA interference microsponges for efficient siRNA delivery. Nature Mater 2012;11:316-22. 